(C) 2000 International Press

Adv. Theor. Math. Phys. 4 (2000) 1093-1147

\title{
Representation of a complex
}

\section{Green function on a real \\ basis: I. General Theory}

Robin Shakeshaft and Bernard Piraux

\author{
Physics Department \\ University of Southern California \\ Los Angeles, CA 90089-0484, USA \\ Laboratoire de Physique Atomique et Moleculaire \\ 2 Chemin du Cyclotron \\ B-1348 Louvain-la-Neuve, Belgium
}

\begin{abstract}
When the Hamiltonian $H$ of a system is represented by a finite matrix $\underline{H}$, constructed from a discrete basis with overlap matrix $\underline{B}$, the matrix representation $\underline{G}(E) \equiv 1 /(E \underline{B}-\underline{H})$ of the resolvent $G(E)=1 /(E-H)$ covers only one branch of $G(E)$. We show how all branches can be specified by the phase $\phi$ of a complex unit of time $t_{\phi} \equiv t_{0} e^{i \phi}$. This permits $\underline{H}$ to be constructed on a real basis; the only duty of the basis is
\end{abstract}

e-print archive: http://xxx.lanl.gov/physics/9909028 
to span the dynamical region of space, without regard for the particular asymptotic boundary conditions that pertain to the problem of interest. Specifically, we show that $P_{\text {cont }} G(E)$, where $P_{\text {cont }}$ projects onto the continuous spectrum of $H$, has the series representation

$$
P_{\text {cont }} G(E)=P_{\text {cont }} e^{-t_{\phi} H}\left(\frac{1}{E}+2 i t_{\phi}^{2} H \sum_{n=1}^{\infty} \frac{1}{n} \mathcal{I}_{n}\left(E t_{\phi}\right) L_{n-1}^{(1)}\left(2 t_{\phi} H\right)\right),
$$

where both the associated Laguerre polynomials, $L_{n-1}^{(1)}\left(2 t_{\phi} H\right)$, and the coefficients $\mathcal{I}_{n}\left(E t_{\phi}\right)$, satisfy 3 -term recurrence relations.

\section{Introduction}

The theoretical treatment of a continuous stationary or quasistationary process requires some knowledge of the resolvent $G(E) \equiv 1 /(E-H)$, where $H$ is the Hamiltonian of the system at hand. There are numerous methods for evaluating $G(E)$, at least approximately. Frequently $H$ is approximated by a finite-dimensional matrix $\underline{H}$. The straightforward substitution of $\underline{H}$ for $H$ leads to the approximation of $G(E)$ by the matrix $\underline{G}(E)=1 /(E \underline{B}-\underline{H})$ where $\underline{B}$ is the overlap matrix for the discrete basis functions used in constructing $\underline{H}$. In principle, the basis functions are only required to accurately span the (generally finite) spatial region where the dynamics take place. However, in practice, unless the wavefunction is matched to its asymptotic form outside the dynamical region, as is done for example in the R-matrix method,[4] the basis may also be burdened by the requirement that it incorporate the asymptotic boundary condition. This is evident from the absence of branch points in $\underline{G}(E)$; the expression $1 /(E \underline{B}-\underline{H})$ has only poles (since the eigenvalue spectrum of $\underline{H}$ is discrete). Some of these poles (those in the bound-state region) are legitimate, while the others (those in the scattering-state region) simulate the branch cut of $G(E)$ by a sequence of discrete points. Hence $\underline{G}(E)$ represents, at best, only one of the branches of the exact resolvent, and this particular branch is determined by the choice of basis.

In this paper we derive a series representation of the resolvent which is such that the asymptotic boundary condition can be imposed independently of the basis, without matching the wavefunction; a particular 
branch of $G(E)$ is specified by the phase of a complex unit of time. In a companion paper [15] we resum this series to give an integral representation of $G(E)$, which is perhaps simpler to use in numerical applications. Both the series and integral representations can be used even when the Hamiltonian is approximated by a real Hermitian matrix $\underline{H}$, constructed from a real basis. This flexibility may permit a real basis to be used more generally and efficiently than otherwise possible, and, in addition, it may prove to be useful in the development and practical realization of a general theory of continuum processes in which detailed knowledge of the asymptotic form of the wavefunction is unnecessary. Recently a reformulation of perturbation theory for multiphoton processes was given [16] which allows, at least in principle, rates and branching ratios to be calculated in terms of the flux through a large hypersphere, without input on the asymptotic form of the wavefunction. However, the practical efficiency of this reformulation depends on the availability of a tractable method for representing the resolvent without explicit reference to the asymptotic boundary condition.

We pause for a moment to mention some of the difficulties that arise when a discrete basis is required to simulate the asymptotic behavior of the open channels of a system. For the sake of discussion we consider a specific but typical basis that is used to construct the Hamiltonian of a one-electron system such as a hydrogen atom; the basis functions are

$$
\sqrt{-2 i \kappa}(2 i \kappa r)^{l+1} e^{i \kappa r} P_{n}(2 i \kappa r) Y_{l m}(\hat{\mathbf{x}})
$$

where $P_{n}(x)$ is a polynomial of degree $n$, where $Y_{l m}(\hat{\mathbf{x}})$ is a spherical harmonic, and where $\kappa$ is a parameter - the wavenumber of the basis. This basis can be readily generalized to a multiparticle system by specifying the orientation of the system in terms of Euler angles and by introducing products of radial functions, one in each of the interparticle distances (or combinations of them), perhaps associating a different wavenumber with each distance. A bound-state wavefunction satisfies a real damped-wave asymptotic boundary condition, which can be described by choosing a real basis with positive pure imaginary wavenumbers. Hence a real basis is well-suited to the description of closed channels and to the study of bound-state properties.[12] However, when $\underline{G}(E)$ is constructed from a real basis it has spurious poles on the "unitarity" branch cut of $G(E)$ along the positive real energy axis, and these poles give rise to spurious resonances with zero width in 
the scattering-state energy region. The wavefunction for a compound (metastable) state satisfies a complex outgoing-wave asymptotic boundary condition, which can be described by choosing a complex basis with wavenumbers lying in the upper-right quadrant of the wavenumber plane; ${ }^{1}$ since the factor $e^{i \kappa r}$ behaves as a damped outgoing-wave, this basis is well suited to the description of both closed channels and outgoing-wave open channels, and can be used to treat half-collision process, e.g., autoionization [3] or photoionization [9]. Furthermore, $\underline{G}(E)$ now has poles in the scattering-state region that lie in the upper half of the complex energy plane, and they simulate a branch cut along a line which is distinct from the real axis, so spurious resonances are only of minor significance. However, a price is paid for choosing the basis to be complex, i.e., $\underline{H}$ is nonHermitian, and therefore unitarity is only approximately satisfied. In fact, the norm $\langle\Psi \mid \Psi\rangle$ of a state vector $|\Psi\rangle$ is a nonanalytic function of $\kappa$ since the bra $\langle\Psi|$ depends on $\kappa^{*}$; consequently, the norm does not converge as the basis size is increased. In addition, it is difficult to extract partial rates, i.e., branching ratios, since a partial rate is also nonanalytic in $\kappa$. Finally, a complex basis cannot, in general, describe the real standing-wave boundary condition satisfied by the wavefunction for a full-collision process. ${ }^{2}$

While a real basis, with pure imaginary wavenumbers, can describe a standing-wave boundary condition, the branch of $G(E)$ cannot be specified uniquely by real basis functions since they do not distinguish between ingoing- and outgoing-wave behavior. On the other hand, when a real basis is used in conjunction with the series and integral representations developed here, the only duty of the basis is to span the dynamical region of space. The only purpose of the purely decaying exponential factor $e^{i \kappa r}$ ( $\kappa$ is positive pure imaginary) is to restrict the range of the basis to the dynamical region.

\footnotetext{
${ }^{1}$ Choosing the basis wavenumbers to lie in the upper right quadrant of the wavenumber-plane is equivalent to rotating the particle coordinates into the lower right quadrant of the complex position-coordinate-plane. See e.g., [14].

${ }^{2}$ An exception occurs when the scattering potential falls off at least as fast as an exponential potential. In this case the deviation of the wavefunction from a standing wave is inconsequential at large distances. A wavefunction that is a real standing wave over short distances has a finite number of nodes, and it can be constructed from a complex basis provided that polynomials $P_{n}(2 i \kappa r)$ of sufficiently high degree are included. At the same time the correct branch of $\underline{G}(E)$ is guaranteed by the factor $e^{i \kappa r}$. See e.g., [2].
} 
In order to impose asymptotic boundary conditions that are independent of the basis we start from the observation that all the particles in a system evolve according to a common time, and the asymptotic behaviour of the system's wavefunction emerges from the initial boundary conditions, at the start of the system's evolution, say time $t=0$. To simplify our analysis we consider a system comprised of only one particle; the generalization to more than one particle is fairly straightforward and will be dealt with elsewhere. The temporal behavior is governed by the time-evolution operator $U(t) \equiv e^{-i H t}$ (we set $\hbar=1$ throughout). Let us introduce the dimensionless variable $\tau \equiv t / t_{0}$, where $t_{0}$ is the unit of time that characterizes the time scale on which the motion of the system occurs. If $\operatorname{Im} E>0$ we can represent $G(E)$ by an integral along the positive $\tau$-axis:

$$
G(E)=-i t_{0} \int_{0}^{\infty} d \tau e^{i\left(t_{0} E\right) \tau} e^{-i\left(t_{0} H\right) \tau}
$$

We can analytically continue this representation of $G(E)$ to a sector of the $E$-plane that includes the negative real $E$-axis if we first project out the bound states, i.e., if we remove the bound-state poles of $G(E)$. Thus we consider $P_{\text {cont }} G(E) P_{\text {cont }}$, where $P_{\text {cont }}$ is the projection operator $P_{\text {cont }}=1-P_{\mathrm{bd}}$, with $P_{\mathrm{bd}}$ the bound-state projection operator. We rotate the contour of integration from the positive real $\tau$-axis through a small angle into the lower-right quadrant of the complex $\tau$-plane, and thereby obtain a representation of $P_{\text {cont }} G(E) P_{\text {cont }}$ which is valid in a region including the negative real $E$-axis. We can represent $G(E)$ throughout the lower half of the $E$-plane by an integral along the negative $\tau$-axis:

$$
G(E)=-i t_{0} \int_{0}^{-\infty} d \tau e^{i\left(t_{0} E\right) \tau} e^{-i\left(t_{0} H\right) \tau}
$$

and again by considering $P_{\text {cont }} G(E) P_{\text {cont }}$, and rotating the integration contour through a small angle (now into the lower-left quadrant of the $\tau$-plane) we obtain a representation of $P_{\text {cont }} G(E) P_{\text {cont }}$ which is valid in a sector of the $E$-plane that also includes the negative real $E$-axis. As we let $E$ approach the positive real axis these two different representations yield two different branches of $P_{\text {cont }} G(E) P_{\text {cont }}$, and since both representations give the same "physical" branch of $P_{\text {cont }} G(E) P_{\text {cont }}$ on the negative real $E$-axis we can define a global representation of $P_{\text {cont }} G(E) P_{\text {cont }}$ on the first - the "physical" — sheet of the Riemann energy surface 
by using Eq. (1) for $\operatorname{Im} E \geq 0$ and Eq. (2) for $\operatorname{Im} E \leq 0$. The point is: A particular branch of $P_{\text {cont }} G(E) P_{\text {cont }}$ on the positive energy axis can be specified by the contour of the global integral representation. Our goal is to perform the integration over $\tau$ in such a way that a signature of the contour is preserved.

That signature is the angle of rotation of the contour, whose vestige is the phase $\phi$ of a complex unit of time $t_{\phi} \equiv t_{0} e^{i \phi}$. The main result of our paper is that the resolvent, with the bound-states removed, can be expressed generally as the series

$$
P_{\text {cont }} G(E)=P_{\text {cont }} e^{-t_{\phi} H}\left(\frac{1}{E}+2 i t_{\phi}^{2} H \sum_{n=1}^{\infty} \frac{1}{n} \mathcal{I}_{n}\left(E t_{\phi}\right) L_{n-1}^{(1)}\left(2 t_{\phi} H\right)\right),
$$

where $L_{n-1}^{(1)}\left(2 t_{\phi} H\right)$ is an associated Laguerre polynomial of degree $n-1$ in the operator $2 t_{\phi} H$, and where the coefficient $\mathcal{I}_{n}\left(E t_{\phi}\right)$ is a number defined by the integral

$$
\mathcal{I}_{n}(a) \equiv \int_{0}^{\infty} d \tau e^{i a \tau}\left(\frac{\tau+i}{\tau-i}\right)^{n} .
$$

The energy, $E$, can take on any value on the first sheet of the Riemann surface cut along the line arg $(E)=-\phi$. The whole Riemann surface can be covered by rotating the cut, i.e., by varying the phase $\phi$. The magnitude, $t_{0}$, of the unit of time, $t_{\phi}$, is a parameter whose value is to some extent, but not entirely, arbitrary. It should not differ greatly from the characteristic unit of time for the motion of the system, i.e., from the time it takes for the wavefunction of the system to change appreciably, for otherwise convergence of the series would be difficult to attain. In particular, $t_{0}$ should not exceed the characteristic duration of the process under study, since a measurable process cannot take place in a time less than it takes for the wavefunction to evolve appreciably. For example, the characteristic duration of an elastic collision process at energy $E$ is of order $1 / E$ so that in this case we require $E t_{0}<1$. For asymptotically large values of $E$ the time-scale is infinitesimally short, i.e., $t_{\phi} \sim 0$, and only the first term on the right side of Eq. (3) contributes, giving $G(E) \sim 1 / E$ for all branches. Apart from this $1 / E$ term, the dependence of $G(E)$ on $E$ is contained in the coefficients $\mathcal{I}_{n}\left(E t_{\phi}\right)$, and a particular branch of $G(E)$ is fixed by these coefficients after specifying the phase $\phi$. The physical branch of $G(E)$, which has 
outgoing-wave behavior, is specified by choosing $\phi$ to be in the range $0<\phi<\pi$, and the other, "unphysical", branch, which has ingoingwave behavior, is specified by choosing $\phi$ to be in the range $0>\phi>-\pi$. In principle, the right side of Eq. (3) should not vary as $t_{\phi}$ varies over its allowed range, but in practice, when the series is truncated and the summation not fully converged, it does vary - the less so, the better the convergence. The presence of $P_{\text {cont }}$ in Eq. (3) is perhaps not surprising since we were obliged to project out the bound states before writing down a global integral representation of the resolvent. [Actually, since $G(E)$ acts, in general, on a wavepacket, we do not need to multiply the Hamiltonian by $P_{\text {cont }}$; rather, we need only let $P_{\text {cont }}$ act on the wavepacket.] When the Hamiltonian contains a potential that has an attractive Coulomb tail, an infinite number of bound states accumulate at threshold, and it is neither necessary nor desirable to remove those bound states just below threshold; we elaborate further on this in sections 2 and 3.

Note that the Laguerre polynomials satisfy a 3-term recurrence relation, and therefore they can be calculated recursively with only a minimal number of multiplications of $\underline{H}$. In addition, as shown in Appendix $\mathrm{C}$, the coefficients $\mathcal{I}_{n}\left(E t_{\phi}\right)$ can be calculated using a 3-term recurrence relation. Furthermore, since the evolution operator $e^{-t_{\phi} H}$ acts for only one unit of time, it can be calculated using a Padé approximant - see Appendix C.

In the derivation of Eq. (3) which we give in section 4, we initially restrict the phase of $t_{\phi}$ to the range $-\pi / 2<\phi<\pi / 2$. However, the series can be analytically continued outside this range. The preliminary restriction on the phase is related to a restriction on the contour of the global integral representation, as we now explain. For the sake of discussion, assume $E$ to have a physically realizeable value, one appropriate to a collision process, i.e., $E$ real and positive. The integrals of Eqs. (1) and (2) differ only by their contours. These contours can be rotated from the positive and negative real $t$-axes into the upper half of the $t$-plane, but they must remain distinct since the integrals represent two different branches of $G(E)$. Hence if we choose one contour to lie along the ray $\arg (t)=\phi$ in the upper-right quadrant, with $\phi$ therefore restricted to $0 \leq \phi<\pi / 2$, we should choose the other contour to lie along the ray $\arg (t)=\pi+\phi$ in the upper-left quadrant, with $\phi$ restricted to $-\pi / 2<\phi \leq 0$; therein lies our preliminary restriction on 
the phase.

To gain an intuitive understanding of the series, we give now a brief heuristic derivation of Eq. (3). We start by formally expressing $G(E)$ as a Taylor series in $H / E$ :

$$
G(E)=\frac{1}{E}+\frac{1}{E} \sum_{n=1}^{\infty}\left(\frac{H}{E}\right)^{n}
$$

This series is an asymptotic series in powers of $H / E$. It is applicable for large $E$, i.e., it is applicable on a short time-scale, but not in all sectors of the complex $E$-plane. In fact, it is not applicable when $E$ is real and positive; for if $H$ is also positive, i.e., if $H$ acts on those of its eigenvectors belonging to the continuous eigenvalue spectrum - whose threshold we choose to be zero - all of the terms $(H / E)^{n}$ are positive and the sum steadily diverges as more terms are included. Hence, for $E$ real and positive we are motivated to delete continuous eigenvalues much larger than $E$ by multiplying the right side of Eq. (5) by a cutoff factor. A natural choice for the cutoff factor is $e^{-t_{\phi} H}$, provided the following restrictions are imposed on $t_{\phi}$ : In order for $e^{-t_{\phi} H}$ to cut off the sum we require that $\operatorname{Re} t_{\phi}>0$, i.e., $|\phi|<\pi / 2$, but since we do not need (or want) to delete continuous eigenvalues much smaller than $E$ we require that $E t_{0}<1$; these are the same restrictions on $t_{\phi}$ mentioned above. (We note again that the restriction on $\phi$ can be lifted by analytic continuation.) We can regather the terms in the sum on the right side of Eq. (5), now modified by the cutoff factor, to yield an expansion in the basis functions $x e^{-x / 2} L_{n}^{(\nu)}(x)$, where $x=2 t_{\phi} H$ and where the $L_{n}^{(\nu)}(x)$ are associated Laguerre polynomials. These basis functions satisfy the end-point boundary conditions for the modified sum, i.e., they vanish linearly at $x=0$ and exponentially at $x=\infty$. Furthermore, these basis functions form a complete set over the interval $0 \leq x<\infty$, and they satisfy the orthogonality relation

$$
\int_{0}^{\infty} d x x^{\nu} e^{-x} L_{m}^{(\nu)}(x) L_{n}^{(\nu)}(x)=\frac{\Gamma(\nu+n+1)}{\Gamma(n+1)} \delta_{m n} .
$$

Here $x$ is understood to be a continuous variable, and consequently we must restrict the operator $H$ to act only on those of its eigenvectors belonging to the continuous eigenvalue spectrum. In other words, we must exclude eigenvectors belonging to the discrete eigenvalue spectrum. Choosing the upper index $\nu$ of the Laguerre polynomials to be 
1 , it follows that

$$
\frac{P_{\text {cont }}}{E-H}=P_{\text {cont }} e^{-t_{\phi} H}\left(\frac{1}{E}+i t_{\phi}\left(2 t_{\phi} H\right) \sum_{n=1}^{\infty} \frac{1}{n} \mathcal{I}_{n}\left(E t_{\phi}\right) L_{n-1}^{(1)}\left(2 t_{\phi} H\right)\right)
$$

where the coefficients, determined using Eq. (6), are

$$
\mathcal{I}_{n}\left(E t_{\phi}\right)=-2 i \int_{0}^{\infty} d H e^{-t_{\phi} H}\left(\frac{1}{E-H}-\frac{e^{-t_{\phi} H}}{E}\right) L_{n-1}^{(1)}\left(2 t_{\phi} H\right),
$$

where here $H$ is understood to be a nonnegative continuous variable of integration. Thereby we arrive at Eq. (3). While not obvious, the coefficients defined by Eq. (8) are the same as those defined by Eq. (4); we prove this in Appendix A. Although Eq. (7) was just derived for $E$ real and positive, the results can be analytically continued to complex E.

It is time to remark on other polynomial expansions of the resolvent. If the Hamiltonian is represented by a finite matrix, whose minimum and maximum eigenvalues are $E_{\min }$ and $E_{\max }$, respectively, one can, by fiat, expand the matrix representation of the resolvent in any set of orthogonal polynomials that form a complete set over a finite interval rescaled to the interval $\left[E_{\min }, E_{\max }\right]$. The most popular of these expansions uses Chebyshev or Faber polynomials $[8,7]$ since the evolution operator $U(t) \equiv e^{-i H t}$ can be expanded very effectively over a finite time interval in terms of these polynomials.[7, 17] The Chebyshev expansion has been remarkably successful in obtaining properties of bound and compound states. [10] However, the Chebyshev and Faber expansions do not discriminate between the discrete and continuum parts of the spectrum of $H$, and notwithstanding some numerical evidence that these expansions can yield reasonably accurate estimates of scattering amplitudes in some simple cases, $[8,7]$ it is unclear at this stage how well-suited are these expansions for the treatment of general collision processes, particularly when the Coulomb tail plays an important role. The long-time behavior of the temporal correlation amplitude (see below) is determined by the spectral density at the threshold of the continuous spectrum. The success of the Chebyshev expansion in dealing with discrete (bound and compound) states can be understood by observing that each of these states decays exponentially with increasing time in a wide sector of the complex time-plane; hence 
the effective time-interval over which $U(t)$ must be expanded is relatively short. However, in a continuum process, wavepacket spreading occurs, and its asymptotic temporal behavior is $1 / t^{3 / 2}$ (for short-range potentials), a rather slow fall-off with $t$.

Our primary interest is in stationary processes in the continuum, for which $E$ is real and positive. We focus in the present paper on the general theory underlying the series representation of the resolvent. As noted already, for simplicity we consider a system comprised of only one particle. Our analysis is largely informal (a mathematically rigorous treatment is, anyway, beyond our abilities). In a companion paper [15] we derive the integral representation, and we give numerical illustrations for the examples of photoionization of a hydrogen atom and s-wave scattering from a $1 /(1+r)^{4}$ potential. We plan to report on an application to a multiparticle system in the future.

We frame our paper within the context of the inclusive rate at which a continuous stationary or quasistationary process occurs. This rate can be expressed in terms of a matrix element of $G(E)$ of the form $\langle\psi|G(E)| \psi\rangle$ where $|\psi\rangle$ is a localized wavepacket, [6]. This matrix element can in turn be expressed, according to Eq. (1), as $-i \int_{0}^{\infty} d t e^{i E t} C(t)$ where $C(t)$ is the temporal correlation amplitude:

$$
C(t) \equiv\langle\psi \mid \psi(t)\rangle
$$

with $|\psi(t)\rangle \equiv U(t)|\psi\rangle$. In the next section we explore the analytic properties of the correlation amplitude in the complex-time-plane. We find that the singularities of $C(t)$ lie in the upper half of the $t$-plane. While we can expand $U(t)$, and thereby $C(t)$, in powers of $t$, the power series for $C(t)$ has only a small (maybe infinitesimal) radius of convergence. In section 3 we transform variables, from $t$ to a variable $u$, to obtain a power series which is more useful. Thus we make a conformal transformation which maps a singularity-free region of the $t$-plane - namely, the half of the complex $t$-plane that lies below the line $\operatorname{Im} t / \operatorname{Re} t=\tan \phi$ - into the unit circle in the $u$-plane. We can express $U(t)$ and $C(t)$ as power series in $u$, with coefficients $c_{n}$ that are rather simply related to the coefficients of the power series in $t$. We analyse the properties, in particular, the large- $n$ behavior, of the coefficients $c_{n}$, though we defer some of the analysis to Appendix D. In section 4 we perform the integration over $t$ and obtain the series representation, Eq. (3), of $G(E)$. We analyse the convergence properties of this series, and also 
show that the higher terms in the series can be resummed as another series that converges rapidly. In Appendix A we prove the equivalence of the two forms of $\mathcal{I}_{n}(a)$ introduced above. In Appendix B we use the series representation of the resolvent to reproduce some known formal results, for example, threshold laws. In Appendix $\mathrm{C}$ we describe some algorithms that are useful for the implementation of the series, and in Appendix $\mathrm{D}$ we analyse the large- $n$ behavior of the coefficients $c_{n}$ when the potential has a Coulomb tail.

\section{Analytic Properties of the Correlation Amplitude}

Recall that $|\psi\rangle$ is a normalizeable, localized wavepacket which evolves in time $t$ as $|\psi(t)\rangle=U(t)|\psi\rangle$, where $U(t) \equiv e^{-i H t}$ is the time-evolution operator and where $H$ is the Hamiltonian of a system that is comprised of only one particle, of mass $\mu$ say. We assume that the wavepacket $|\psi\rangle$ includes a continuous superposition of scattering eigenstates of $H$, and is not merely a superposition of discrete (bound- and compound) eigenstates. If the potential has an attractive long-range Coulomb tail, a localized wavepacket has, in general, a nonzero overlap with an infinite number of bound-eigenstates of $H$. Indeed, if $L$ is the characteristic linear dimension of $|\psi\rangle$ in position space, Rydberg bound states with energy eigenvalues greater than or of the order of $-\mu / L$ are indistinguishable in the composition of the wavepacket from scattering eigenstates with energy eigenvalues less than or of the order of $\mu / L$. As a consequence, high Rydberg bound states, through their contribution to the correlation function, play an important role in continuum processes, a feature we observe below. The case where the potential has a Coulomb tail is usually an exception requiring special treatment. Leaving aside this case for the moment, the continuum portion of $|\psi(t)\rangle$ spreads linearly in time, and therefore occupies a volume (in 3-dimensional position space) that is proportional to $t^{3}$. Since $U(t)$ is unitary, $\langle\psi(t) \mid \psi(t)\rangle$ is conserved in time, and so the continuum portion of the wavepacket attenuates at each point in space as $t^{-3 / 2}$. Consequently, the correlation amplitude $C(t) \equiv\langle\psi \mid \psi(t)\rangle$ contains a component which vanishes as $t^{-3 / 2}$ for $t \sim \infty$. Therefore $C(t)$ has a branch point at infinity, and since this branch point is of order two there 
must be another branch point, joined to the branch point at infinity by a cut in the complex $t$-plane.

As an example, consider a free particle (of mass $\mu$ ) whose position is initially described by the Gaussian wavepacket

$$
\langle\mathbf{x} \mid \psi\rangle=\left(\kappa_{0}^{2} / \pi\right)^{3 / 4} e^{-\frac{1}{2} \kappa_{0}^{2} r^{2}} .
$$

The Hamiltonian governing the evolution of the wavepacket is the kinetic energy operator $-(1 / 2 \mu) \nabla^{2}$; we find that

$$
C(t)=\left(\frac{2}{2+i t / t_{0}}\right)^{3 / 2}
$$

where we recall that $t_{0}$ characterizes the time scale for the evolution of the wavepacket $|\psi\rangle$, and is defined in the present example as $t_{0}=\mu / \kappa_{0}^{2}$. Note that $C(t)$ has branch points at $t=2 i t_{0}$ and $t=\infty$. At time $t=0$ the wavepacket has a characteristic width in position space of $1 / \kappa_{0}$, but since the wavepacket has a momentum distribution of width $\kappa_{0}$ its spatial spread after time $t$ is $\kappa_{0} t / \mu$, and this spread exceeds the original width of the wavepacket when $t$ is comaparable to $t_{0}$. In other words, the singularity at $2 i t_{0}$ is a signature of the time at which the wavepacket becomes significantly deformed by spreading. The wavepacket can evolve either forwards or backwards in time to the single point $t=\infty$, and the result depends on the arrow of time. Hence the wavepacket is double-valued at $t=\infty$, a property that is encompassed by the branch point at $t=\infty$.

A general (nonGaussian) wavepacket has a momentum distribution whose very-high-momentum components are appreciable, and the spatial tail of the wavepacket deforms the moment it begins to evolve freely. Even the tail of a Gaussian wavepacket deforms instantly if it evolves under the influence of a potential that can transfer a large momentum to the particle. Hence, in general $t_{0}=0$, i.e., the correlation amplitude has a branch point singularity at the origin. To be more concrete, suppose that $H$ has bound-state and scattering-state eigenvectors $\left|\chi_{\mathrm{bd}, n}\right\rangle$ and $\left|\chi_{\mathbf{k}}\right\rangle$, respectively, with real energy eigenvalues $E_{\mathrm{bd}, n}$ and $E_{k} \equiv k^{2} / 2 \mu$, respectively. We can express $|\psi\rangle$ as the superposition

$$
|\psi\rangle=\sum_{n} \psi_{\mathrm{bd}, n}\left|\chi_{\mathrm{bd}, n}\right\rangle+\int d^{3} k \psi(\mathbf{k})\left|\chi_{\mathbf{k}}\right\rangle,
$$


where the eigenvectors are normalized so that $\psi_{\mathrm{bd}, n}=\left\langle\chi_{\mathrm{bd}, n} \mid \psi\right\rangle$ and $\psi(\mathbf{k})=\left\langle\chi_{\mathbf{k}} \mid \psi\right\rangle$. This superposition evolves in time as

$$
|\psi(t)\rangle=\sum_{n} \psi_{\mathrm{bd}, n}\left|\chi_{\mathrm{bd}, n}\right\rangle e^{-i E_{\mathrm{bd}, n} t}+\int d^{3} k \psi(\mathbf{k}) e^{-i E_{k} t}\left|\chi_{\mathbf{k}}\right\rangle,
$$

and the correlation amplitude is

$$
C(t)=C_{\mathrm{bd}}(t)+C_{\text {cont }}(t)
$$

where

$$
\begin{aligned}
C_{\mathrm{bd}}(t) & =\sum_{n}\left|\psi_{\mathrm{bd}, n}\right|^{2} e^{-i E_{\mathrm{bd}, n} t} \\
C_{\mathrm{cont}}(t) & =\int d^{3} k|\psi(\mathbf{k})|^{2} e^{-i E_{k} t}
\end{aligned}
$$

We can let $t$ move into the lower-half complex $t$-plane; the exponential $e^{-i E_{k} t}$ decays with increasing $k$ and both $C_{\mathrm{bd}}(t)$ and $C_{\text {cont }}(t)$ are welldefined. However, $e^{-i E_{\mathrm{bd}, n} t}$ explodes as $t \rightarrow \infty$ in the lower-half of the $t$-plane; hence $C_{\mathrm{bd}}(t)$ has an essential singularity at $t=\infty$ and is unbounded. Furthermore, if we allow $t$ to move into the upper-half complex $t$-plane, $e^{-i E_{k} t}$ explodes with increasing $k$, as the Gaussian $\exp \left(E_{k} \operatorname{Im} t\right)$. Therefore, unless $|\psi(\mathbf{k})|^{2}$ decreases more rapidly than this, $C_{\text {cont }}(t)$ is formally undefined in the upper-half of the $t$-plane. Suppose for the moment that $|\psi(\mathbf{k})|$ were to decrease with increasing $k$ as $e^{-E_{k} t_{0}}$. In this case $C_{\text {cont }}(t)$ would be formally defined in the region $\operatorname{Im} t<2 t_{0}$. However, by rotating the contour of $k$-integration through an angle $\Theta$ into the first octant of the lower-right quadrant of the $k$ plane, assuming that $|\psi(\mathbf{k})|$ is free of singularities in this octant, we can analytically continue $C_{\text {cont }}(t)$ throughout any finite region of the sector $0 \leq \arg (t)<2 \Theta$ in the upper-right quadrant of the $t$-plane, excluding the section of the positive imaginary $t$-axis above $2 i t_{0}$ since both $e^{-E_{k} t_{0}}$ and $e^{-i E_{k} t}$ are undamped oscillatory functions of $k$ when $\Theta=\pi / 4$ and $t$ is pure imaginary. Therefore $C_{\text {cont }}(t)$, and hence $C(t)$, are analytic in both the lower- and right-half $t$-planes, but they have branch points at $2 i t_{0}$, and branch cuts extending from $2 i t_{0}$ to infinity. In general, $|\psi(\mathbf{k})|$ decreases as a power of $1 / k$ with increasing $k$, less rapidly than a Gaussian, and we may expect $C(t)$ to have a branch point at the origin, i.e., a branch point at $2 i t_{0}$ with $t_{0} \rightarrow 0$. Furthermore, $|\psi(\mathbf{k})|$ generally has singularities at finite points in the complex $k$-plane (in 
contrast, $e^{-E_{k} t_{0}}$ has an essential singularity at $k=\infty$ ); but if there were no singularities in the lower-right quadrant of the $k$-plane, we could rotate the contour of $k$-integration so that it runs along the right edge of the negative imaginary axis, and we could subsequently move $t$ from the positive real axis through the upper-half $t$-plane to the upper edge of the negative real axis. In this case $C(t)$ would be defined everwhere in the finite complex $t$-plane, except possibly for a branch point at the origin, which we cannot exclude unless we can move $t$ continuously along a closed loop around the origin (without discontinuously moving the contour of $k$-integration). Hereafter we formally define $t_{0}$ such that the singularity of $C(t)$ nearest to the origin is located at $2 i t_{0}$, and we draw a cut along the positive imaginary axis from $2 i t_{0}$ to $\infty$. Of course, if, by this definition, $t_{0}$ were to vanish, it could not serve as a unit of time; however, this is not of concern in practice, for in practice $t_{0}$ may be small but it does not vanish, a matter we return to at the end of this section.

To determine the general asymptotic behavior of $C(t)$ for $t \sim \infty$ let us transform the integration variable $\mathbf{k}$ to $\mathbf{k} / \sqrt{t}$ on the right side of Eq. (16). Assuming the potential is short-range, we can, as a first approximation, replace $|\psi(\mathbf{k} / \sqrt{t})|^{2}$ by $|\psi(\mathbf{0})|^{2}$ for $t \sim \infty$. However, if the wavepacket carries orbital angular momentum $|\psi(\mathbf{0})|^{2}$ may vanish; small values of $k$ are inhibited by the centrifugal barrier. If $l$ is the smallest angular momentum quantum number present in the wavepacket, $|\psi(\mathbf{k})|^{2}$ vanishes as $k^{2 l}$ for $k \sim 0$. Therefore we write

$$
|\psi(\mathbf{k})|^{2} \equiv k^{2 l}\left|\psi_{l}(\mathbf{k})\right|^{2} \text {. }
$$

It follows that

$$
C_{\text {cont }}(t) \sim e^{-i(2 l+3) \pi / 4}(2 \pi)^{3 / 2}(2 l+1) ! !\left|\psi_{l}(\mathbf{0})\right|^{2}(\mu / t)^{2 l+3 / 2}, \quad t \sim \infty,
$$

where we used

$$
\int_{0}^{\infty} d k k^{2 m} e^{-a k^{2}}=\frac{(2 m-1) ! !}{2(2 a)^{m}} \sqrt{\frac{\pi}{a}} .
$$

Note that the large- $t$ behavior of $C_{\text {cont }}(t)$ is determined by small $k$, i.e., by the continuum eigenvalues of $H$ that are close to threshold. If the potential has a Coulomb tail $|\psi(\mathbf{0})|^{2}$ is infinite, and we must factor out the divergence arising from the normalization constant. Thus, if 
the Coulomb tail is $-Z e^{2} / r$ at large radial distances $r$, we remove an offending factor from $|\psi(\mathbf{k})|^{2}$ by writing (for all $l$ )

$$
|\psi(\mathbf{k})|^{2} \equiv\left(\frac{2 \pi \gamma}{1-e^{-2 \pi \gamma}}\right)|\tilde{\psi}(\mathbf{k})|^{2}
$$

where $\gamma=Z /\left(a_{0} k\right)$, with $a_{0}=1 / \mu e^{2}$. After making the transformation $\mathbf{k} \rightarrow \mathbf{k} / \sqrt{t}$, we have $\gamma \sim\left(Z / a_{0} k\right) \sqrt{t}$ and the prefactor on the right side of Eq. (19) either vanishes exponentially (if $Z<0$ ) or becomes the divergent function $\left(2 \pi Z / a_{0} k\right) \sqrt{t}$ (if $Z>0$ ) when we let $t$ increase to infinity in any sector excluding the negative real axis. Hence, when an attractive $(Z>0)$ Coulomb tail is present, $C_{\text {cont }}(t)$ falls off only as $1 / t$ :

$$
C_{\text {cont }}(t) \sim-8 i \pi^{2} Z \mu|\tilde{\psi}(\mathbf{0})|^{2} /\left(a_{0} t\right), \quad t \sim \infty \quad(Z>0) .
$$

Note, however, that a Coulomb tail gives rise to an essential singularity at $t=\infty$. (If we were to let $t$ increase to infinity in any sector excluding the negative real axis on the second sheet of the Riemann $t$-surface, $C_{\text {cont }}(t)$ would vanish exponentially if $Z>0$.) A branch point at $t=\infty$ remains, but it does not dominate the asymptotic behavior. Futhermore, if $Z>0$, Rydberg states converging to threshold from below cannot be distinguished in the wavepacket from continuum eigenstates converging to threshold from above, and therefore we have $C_{\mathrm{bd}}(t) \sim C_{\text {cont }}(t)$ for $t$ approaching $\infty$ in the upper-half $t$-plane (the half-plane in which lower-lying bound states decay exponentially). It follows that, in the upper-half $t$-plane,

$$
C(t) \sim-16 i \pi^{2} Z \mu|\tilde{\psi}(\mathbf{0})|^{2} /\left(a_{0} t\right), \quad t \sim \infty \quad(Z>0) .
$$

In other words, we gain a factor of 2 in the asymptotic form of $C(t)$ through the contribution of high Rydberg states. As seen in Eq. (122) of Appendix $\mathrm{B}$, this factor of 2 enters the rate for a continuum process near threshold. When the potential has a Coulomb tail, the sum (over bound states) on the right side of Eq. (15) is convergent, but not uniformly, for $t$ in the upper-half $t$-plane, and we cannot interchange the limit $t \rightarrow \infty$ and the sum. In contrast, when the potential is short-range $C_{\mathrm{bd}}(t) \sim 0$ for $t \sim \infty$ in the upper-half $t$-plane.

To gain further insight into the behavior of $C(t)$ at large $t$ it is useful to express the time-evolution operator $U(t)$ in terms of the resolvent $G(E)$. We have [6]

$$
U(t)=\frac{1}{2 \pi i} \int_{\mathcal{C}} d E e^{-i E t} G(E)
$$




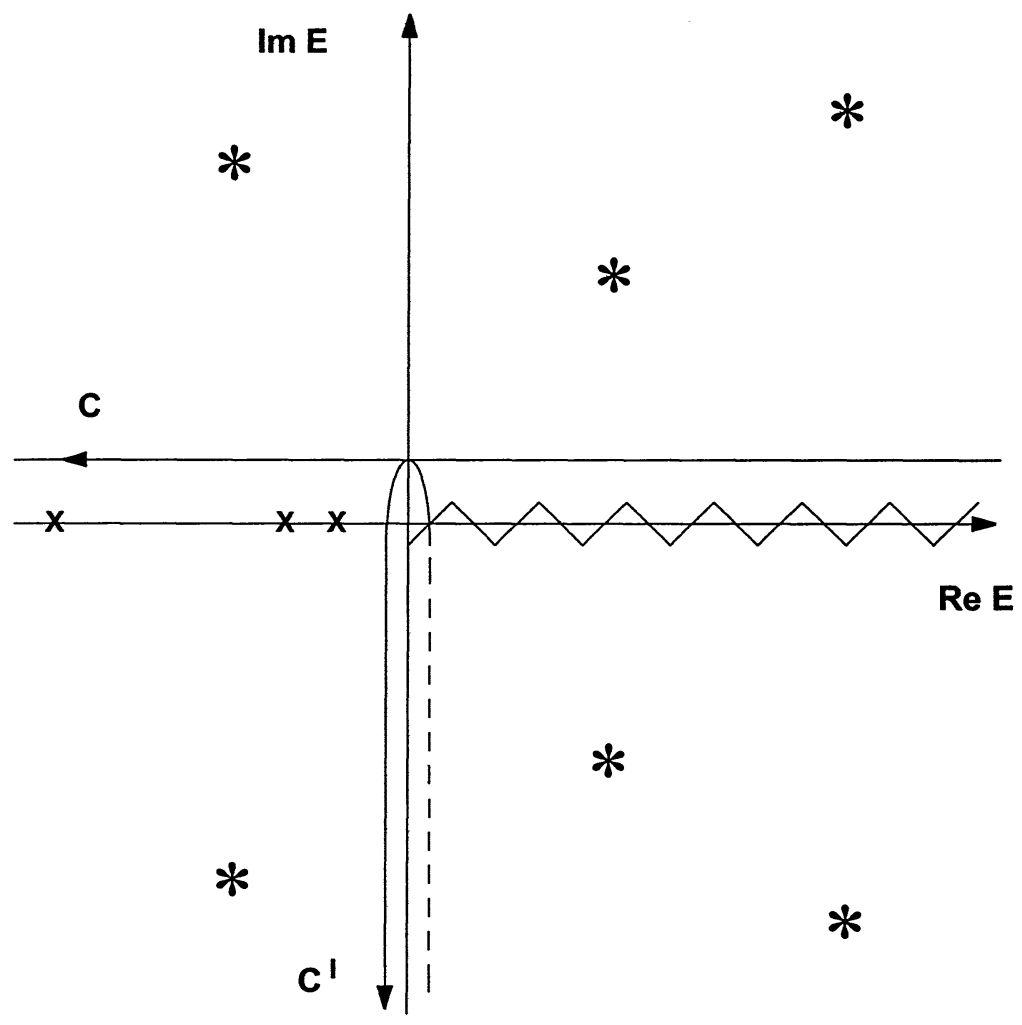

Figure 1: The contour $\mathcal{C}$ runs along the upper edge of the real axis of the physical sheet of the Riemann energy surface cut (the zig-zag line) along the positive real axis (the "unitarity" cut). Bound state poles, indicated by $\times$, lie on the negative real axis of the physical sheet, while resonance poles, indicated by $\star$, lie on the unphysical sheet reached by crossing the cut. The resonance poles are distributed symmetrically about the cut. The contour $\mathcal{C}^{\prime}$ is the result of bending $\mathcal{C}$ around the negative imaginary axis; the left half of $\mathcal{C}^{\prime}$ lies on the physical sheet, while the right half lies on the unphysical sheet. 
where, assuming that $t$ is real and positive, the contour $\mathcal{C}$ runs along the upper edge of the real $E$-axis from $\infty$ to $-\infty$; see Fig. 1 . The resolvent has branch points at $E=0$ and $E=\infty$, and is defined on a two-sheeted Riemann energy surface; the "unitarity" branch cut is drawn along the positive real $E$-axis. In addition to branch points, $G(E)$ has bound-state poles at points $E_{\mathrm{bd}, n}$ on the negative real energy axis of one sheet - the physical sheet - and has resonance poles at points $E_{\text {res }, n}$ and $E_{\text {res }, n}^{*}$ in the lower and upper half-planes, respectively, of the other sheet - the unphysical sheet. Let us bend the contour $\mathcal{C}$ around the branch point at $E=0$, into the lower-half $E$-plane, so that the new contour, $\mathcal{C}^{\prime}$, wraps around the negative imaginary axis. [6] As we distort $\mathcal{C}$ it sweeps over the bound-state poles (on the physical sheet) and also over those resonance poles dispersed in the lower-right quadrant on the unphysical sheet of the energy-plane. It follows that

$$
\begin{aligned}
& |\psi(t)\rangle \\
& =\frac{1}{2 \pi i} \int_{\mathcal{C}} d E e^{-i E t} G(E)|\psi\rangle \\
& =\sum_{n} \psi_{\mathrm{bd}, n}\left|\chi_{\mathrm{bd}, n}\right\rangle e^{-i E_{\mathrm{bd}, n} t}+\sum_{n} \psi_{\mathrm{res}, n}\left|\chi_{\mathrm{res}, n}\right\rangle e^{-i E_{\mathrm{res}, n} t}+\left|\psi_{\mathrm{bg}}(t)\right\rangle
\end{aligned}
$$

where $\left|\chi_{\text {res }, n}\right\rangle$ is an eigenvector of $H$ satisfying outgoing-wave boundary conditions corresponding to a compound (resonance) state ${ }^{3}$ with a complex energy $E_{\text {res }, n}$ whose real part is positive, where $\psi_{\text {res }, n}=\left\langle\chi_{\text {res }, n} \mid \psi\right\rangle$, and where $\left|\psi_{\mathrm{bg}}(t)\right\rangle$ describes the continuum background:

$$
\left|\psi_{\mathrm{bg}}(t)\right\rangle=\frac{1}{2 \pi i} \int_{\mathcal{C}^{\prime}} d E e^{-i E t} G(E)|\psi\rangle .
$$

As expected from our earlier discussion, if the potential is short-range the vector $\left|\psi_{\mathrm{bg}}(t)\right\rangle$ attenuates as $t^{-3 / 2}$ with increasing $t$; a general proof, due to Zumino, is presented in Ref. [6]. In the case where the potential has a Coulomb tail, the behavior of $\left|\psi_{\mathrm{bg}}(t)\right\rangle$ at large $t$ has been analyzed by Dollard.[5] Note that once $\mathcal{C}$ has been deformed to $\mathcal{C}^{\prime}$ we can analytically continue the right side of Eq. (25), and hence $C(t)$, from the real positive $t$-axis to the entire right half of the complex $t$-plane.

\footnotetext{
${ }^{3}$ When $E_{k}$ is complex the continuum eigenfunctions $\left\langle\mathbf{x} \mid \chi_{\mathbf{k}}\right\rangle$ explode exponentially in position space, as $\exp (i \mathbf{k} \cdot \mathbf{x})$, for $|\mathbf{x}| \sim \infty$. Consequently, certain integrals over $\mathbf{x}$ - for example, $\left\langle\psi \mid \chi_{\text {res, } n}\right\rangle$ - are formally undefined. However, such integrals may be defined through analytic continuation, e.g., consider $\int_{0}^{\infty} d r e^{a r}$, which is $-1 / a$ for all $a \neq 0$.
} 
When $t$ is real and negative, $U(t)$ has an integral representation similar to the right side of Eq. (22) but with a contour $\overline{\mathcal{C}}$ running along the lower edge of the real $E$-axis and in the direction opposite to $\mathcal{C}$. Assuming that the Hamiltonian is invariant under time-reversal, the two representations are related through

$$
G\left(E^{*}\right)=K G(E) K^{\dagger}
$$

where $K$ is the antiunitary time-reversal operator.[11] Hence, if $|\bar{\psi}\rangle$ represents the time-reverse of the wavepacket $|\psi\rangle$, i.e., if $|\bar{\psi}\rangle=K|\psi\rangle$, we have, with $t$ real and negative,

$$
\begin{aligned}
|\bar{\psi}(t)\rangle & =\frac{1}{2 \pi i} \int_{\overline{\mathcal{C}}} d E e^{-i E t} G(E)|\bar{\psi}\rangle \\
& =\frac{1}{2 \pi i} \int_{\overline{\mathcal{C}}} d E e^{-i E t} K G\left(E^{*}\right) K^{\dagger}|\bar{\psi}\rangle \\
& =K \frac{1}{2 \pi i} \int_{\mathcal{C}} d E e^{i E t} G\left(E^{*}\right)|\psi\rangle \\
& =K|\psi(-t)\rangle,
\end{aligned}
$$

where in the second step we used Eq. (26) and in the third step we noted that $K^{\dagger} K=1$ and that $K$ complex-conjugates c-numbers. If on the right side of Eq. (27) we deform the integration contour into one wrapped around the positive imaginary $E$-axis, we obtain an expression for $|\bar{\psi}(t)\rangle$ that is similar to the right-side of Eq. (24) but with all terms time-reversed. The time-reversed resonance terms correspond to the conjugate poles of $G(E)$ in the upper-right quadrant of the $E$-plane on the unphysical sheet. Let us introduce the new correlation amplitude

$$
\bar{C}(t) \equiv\langle\bar{\psi} \mid \bar{\psi}(t)\rangle
$$

As long as $t$ is real and negative we can use Eq. (30) to write

$$
\begin{aligned}
\bar{C}(t) & =\langle\bar{\psi}|[K|\psi(-t)\rangle] \\
& =\langle\psi(-t)|\left[K^{\dagger}|\bar{\psi}\rangle\right] \\
& =\langle\psi(-t)|| \psi\rangle \\
& =[C(-t)]^{*},
\end{aligned}
$$

where in the second step we noted [11] that, since $K$ is antilinear, $\langle b|(K|a\rangle)=\langle a|\left(K^{\dagger}|b\rangle\right)$ for any two kets $|a\rangle$ and $|b\rangle$. After deforming the contour $\overline{\mathcal{C}}$, we can analytically continue $\bar{C}(t)$ into the entire left 
half of the complex $t$-plane, and since both $\left[\bar{C}\left(t^{*}\right)\right]^{*}$ and $C(-t)$ are also analytic functions of $t$ in this region we can generalize Eq. (35) to

$$
\left[\bar{C}\left(t^{*}\right)\right]^{*}=C(-t)
$$

for $t$ anywhere in the left half of the complex $t$-plane. When $t$ lies on the negative imaginary axis, $C(t)$ is real, and therefore $\left[C\left(-t^{*}\right)\right]^{*}=$ $C(t)=\left[\bar{C}\left(-t^{*}\right)\right]^{*}$. It follows that $\bar{C}(t)$ is the analytic continuation of $C(t)$ into the left half of the complex $t$-plane. If the point $t$ is moved over the branch cut along the positive imaginary axis, from the left edge of the cut to the right edge, on the same sheet, the correlation amplitude jumps discontinuously from $\bar{C}(t)$ to $C(t)$; in other words, at the cut $\bar{C}(t)$ and $C(t)$ are different branches of the same multivalued correlation amplitude.

To conclude: In general, $C(t)$ is a nonsingular function of $t$ in the right-half of the finite complex $t$-plane, but has a branch point on the positive imaginary axis at $t=2 i t_{0}$. We can expand $C(t)$ in the righthalf of the $t$-plane as - c.f. Eq. (14) -

$$
\begin{aligned}
C(t) & =C_{\mathrm{bd}}(t)+C_{\mathrm{res}}(t)+C_{\mathrm{bg}}(t) \\
& \equiv \sum_{n}\left|\psi_{\mathrm{bd}, n}\right|^{2} e^{-i E_{\mathrm{bd}, n} t}+\sum_{n}\left|\psi_{\mathrm{res}, n}\right|^{2} e^{-i E_{\mathrm{res}, n} t}+\left\langle\psi \mid \psi_{\mathrm{bg}}(t)\right\rangle,
\end{aligned}
$$

where $C_{\mathrm{bg}}(t)$ falls off as $t^{-3 / 2}$ or, if the potential has an attractive Coulomb tail, as $1 / t$ for $t \sim \infty$. If the potential has a Coulomb tail $C_{\mathrm{bg}}(t)$ has an essential singularity at $t=\infty$. Due to the exponential factor(s), $C_{\mathrm{bd}}(t)$ and $C_{\text {res }}(t)$ also have essential singularities at $t=\infty$. In fact, as $t$ increases to infinity in the lower half of the the $t$-plane $C_{\text {bd }}(t)$ explodes exponentially, and $C_{\text {res }}(t)$ exhibits similar behavior for $t$ within some other sector of the $t$-plane. However, $C_{\mathrm{bd}}(t)$ is bounded in the upper-half of the $t$-plane. Also, $C_{\text {res }}(t)$ is bounded in the lowerright quadrant of the $t$-plane since $\operatorname{Re} E_{\text {res }, n}>0$ and $\operatorname{Im} E_{\text {res, } n}<0$. In fact, $C_{\text {res }}(t)$ is bounded in an even wider region since $G(E)$ has no poles within a sector of the $E$-plane, say $-\Xi_{0}<\arg (E)<\Xi_{0}$, which contains the positive real energy axis; therefore $\operatorname{Im} E_{\text {res }, n} t<0$, and $C_{\text {res }}(t)$ is bounded, throughout the sector $0 \leq \arg (t)<\Xi_{0}$ of the $t$-plane. Hence $C(t)$ is bounded throughout the sector $0 \leq \arg (t)<\Xi_{0}$. Similarly, $\bar{C}(t)$ is nonsingular in the left-half of the finite complex $t$-plane, $\bar{C}_{\text {res }}(t)$ is bounded throughout the lower-left quadrant of the $t$-plane, and beyond, and $\bar{C}(t)$ is bounded throughout the sector $\pi-\Xi_{0} \leq \arg (t)<\pi$. We 
can generate a power series for $C(t)$ by expanding $U(t)=e^{-i H t}$ in powers of $t$; we have

$$
|\psi(t)\rangle=\sum_{m=0}^{\infty} \frac{(-i H t)^{m}}{m !}|\psi\rangle,
$$

and therefore introducing the dimensionless variable $\tau=t / t_{0}$, assuming that $t_{0} \neq 0$, we obtain

$$
C(t)=\sum_{m=0}^{\infty}\left\langle\psi\left|\left(-i H t_{0}\right)^{m}\right| \psi\right\rangle \frac{\tau^{m}}{m !} .
$$

The radius of convergence of this expansion of $C(t)$ in powers of $\tau$ is 2 since the singularity of $C(t)$ that is nearest to the origin is, by definition, located at $2 i t_{0}$. Note that we just assumed $t_{0} \neq 0$, yet earlier we remarked that we can have $t_{0}=0$. While in principle it is generally true that $t_{0}=0$, in practice $t_{0} \neq 0$, as we now explain. The time that it takes the wavepacket to deform significantly from its initial form is governed by the highest speed in the velocity distribution of the wavepacket as it evolves in the presence of the potential. Now the highest speed in the velocity distribution is effectively determined by the largest eigenvalue, $E_{\max }$ say, of the smallest matrix $\underline{H}$ that can accurately represent $H$ in the expression $e^{-i H t}|\psi\rangle$. Hence, in practice the characteristic value of $t_{0}$ is $1 / E_{\max }$, which may be very small but is nonzero.

\section{Conformal Transformation}

To calculate the rate for some continuous stationary or quasistationary process from a correlation amplitude we need to know the correlation amplitude for all $t$ on either the positive or negative real axis. However, the power series in $\tau$ is useful only for $0 \leq|t|<2 t_{0}$. Since $C(t)$ is singular on the positive imaginary axis, it is expedient to divide the $t$-plane into two half-planes separated by a line through the origin, and to conformally map the lower of these half-planes (i.e., the one free of singularities) into the unit circle; see Fig. 2. Thus we change variables from $t$ to

$$
u=\frac{\tau+i e^{i \phi}}{\tau-i e^{i \phi}}
$$




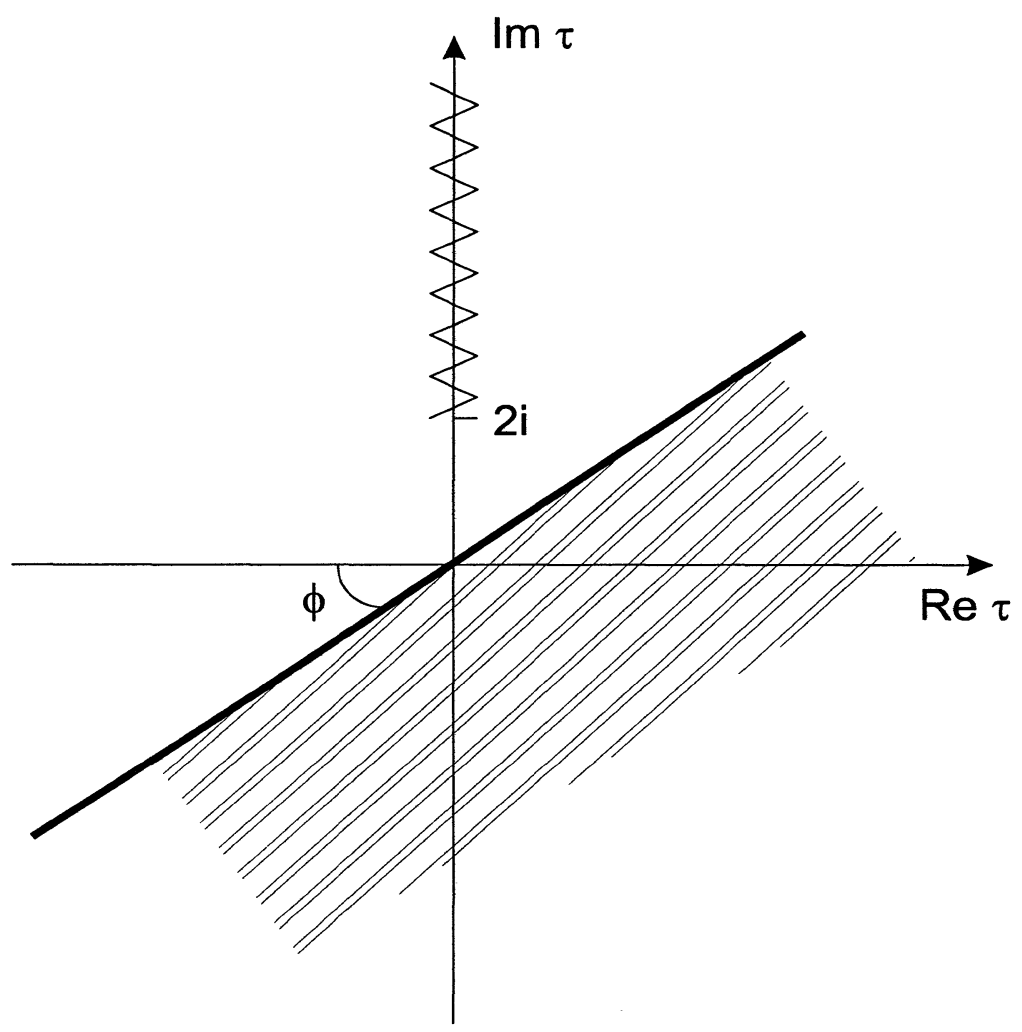

Figure 2: The correlation amplitude $C(t)$ has a branch-point singularity on the positive imaginary axis in the $\tau \equiv t / t_{0}$ plane, and another one at infinity. The unit of time $t_{0}$ is defined so that the singularity nearest the origin is at $\tau=2 i$. We have drawn a cut extending up the positive imaginary axis from $2 i$. One-half (the hatched section) of the $\tau$-plane is conformally mapped onto a unit circle. 
The mapping $u$ depends on both $t$ and the complex unit of time

$$
t_{\phi}=e^{i \phi} t_{0}
$$

and a particular branch of $C(t)$ can be specified by $\phi$, or, rather, a range of values of $\phi$. As $\phi$ varies over the range $-\pi / 2<\phi<\pi / 2$ the boundary line $\operatorname{Im} t / \operatorname{Re} t=\tan \phi$ rotates through one revolution, and we remain on the same branch of $C(t)$. To pass to another branch of $C(t)$ we must allow $\phi$ to move out of this range. We can do this by analytic continuation in the variable $t_{\phi}$, to the left-half of the $t_{\phi}$-plane, i.e., to values of $\phi$ in the range $\pi / 2 \leq|\phi|<\pi$.

Note that the boundary line $\operatorname{Im} t / \operatorname{Re} t=\tan \phi$ is mapped onto the circumference of the unit circle in the $u$-plane, and the real positive (negative) $t$-axis is mapped onto the broken line shown in the upper (lower) semicircle of Fig. 3 if $\phi$ is positive (negative). The points $t=0$, $t=t_{\phi}, t=\infty, t=-i t_{\phi}$, and $t=i t_{\phi}$ are mapped onto the points $u=-1, u=i, u=1, u=0$, and $u=\infty$, respectively. There are no singularities inside the unit circle, and only one on the circumference, at $u=1$ (corresponding to the singularity at $t=\infty$ ). A singularity on the positive imaginary axis of the $t$-plane is mapped onto a point outside the unit circle in the lower (upper) half of the $u$-plane if $\phi$ is positive (negative), and moves onto the real $u$-axis as $\phi$ vanishes.

We now express $C(t)$ as a power series in $u$. Substituting for $\tau$ in the power series on the right side of Eq. (40) using

$$
\tau=-i e^{i \phi}\left(\frac{1+u}{1-u}\right)
$$

and noting that

$$
\left(\frac{1+u}{1-u}\right)^{m}=\sum_{n=0}^{\infty} \frac{1}{n !} P_{n}(m) u^{n},
$$

where $P_{n}(m)$ is the polynomial ${ }^{4}$

$$
P_{n}(m)=\left[\frac{d^{n}}{d u^{n}}\left(\frac{1+u}{1-u}\right)^{m}\right]_{u=0},
$$

${ }^{4}$ Incidentally, it can be shown that

$$
P_{n}(m)=\frac{(m+n-1) !}{(m-1) !}{ }_{2} F_{1}(-n,-m,-m-n+1 ;-1), \quad n \geq 1,
$$

with $P_{0}(m)=1$. The $P_{n}(m)$ are Krawtchouk polynomials, which for $n \geq 1$ satisfy 


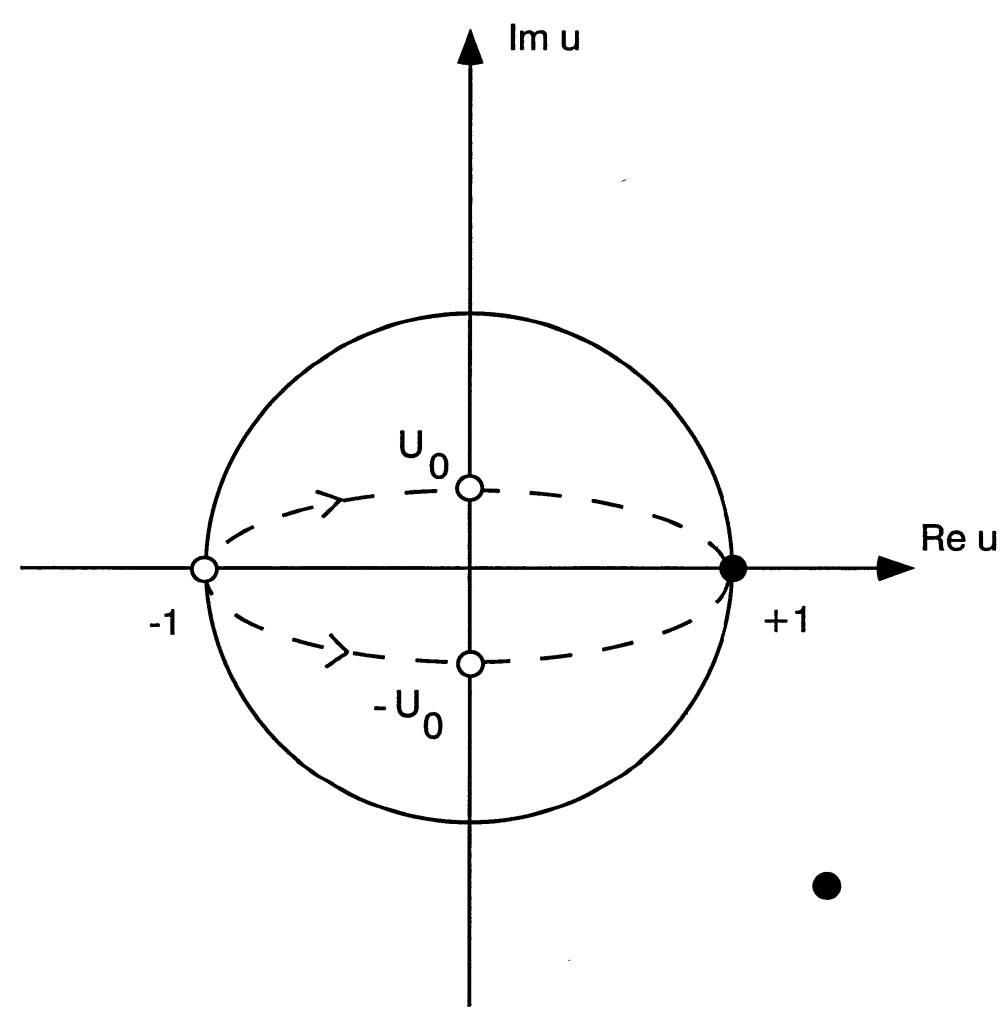

Figure 3: The unit circle in the $u$-plane, where $u=\left(\tau+i e^{i \phi}\right) /\left(\tau-i e^{i \phi}\right)$. If the phase $\phi$ is positive (negative), the real positive (negative) axis in the $\tau$-plane is mapped onto the trajectory indicated by the broken line in the upper (lower) semicircle of the $u$-plane. This trajectory is furthest from the real $u$-axis at the point $u_{0}$ or $-u_{0}$ where $u_{0}=i \cos \phi /(1+$ $\sin |\phi|)$. The correlation amplitude has a singularity (indicated by $\bullet$ ) at $u=1$ and another singularity outside the unit circle. 
we obtain the new power series:

$$
C(t)=\sum_{n=0}^{\infty} c_{n}\left(t_{\phi}\right) u^{n},
$$

where

$$
c_{n}\left(t_{\phi}\right)=\left\langle\psi\left|C_{n}\left(-t_{\phi} H\right)\right| \psi\right\rangle
$$

and where, with $z$ the operator $-t_{\phi} H$,

$$
C_{n}(z)=\sum_{m=0}^{\infty} \frac{P_{n}(m)}{n ! m !} z^{m} .
$$

Since $u=-1$ when $t=0$, and since $C(0)=\langle\psi \mid \psi\rangle$, we have

$$
\sum_{n=0}^{\infty}(-1)^{n} c_{n}\left(t_{\phi}\right)=\langle\psi \mid \psi\rangle
$$

which provides a useful check on the accuracy of the coefficients $c_{n}\left(t_{\phi}\right)$ in a practical application. Since $C(t)$ is nonsingular everywhere inside the unit circle in the $u$-plane, the power series in $u$, on the right side of Eq. (46), converges for all $|u|<1$. The point $u=1$ requires special consideration, as discussed below.

The series for $C_{n}(z)$ can be expressd in closed form. This is obvious when $n=0$ since $P_{0}(m)=1$ and we have

$$
C_{0}(z)=e^{z}
$$

For $n \geq 1$, we use Eqs. (45) and (48) to write:

$$
\begin{aligned}
C_{n}(z) & =\frac{1}{n !}\left\{\frac{d^{n}}{d u^{n}}\left[\sum_{m=0}^{\infty} \frac{z^{m}}{m !}\left(\frac{1+u}{1-u}\right)^{m}\right]\right\}_{u=0}, \\
& =\frac{1}{n !} e^{z}\left[\frac{d^{n}}{d u^{n}} e^{2 z\left(\frac{u}{1-u}\right)}\right]_{u=0},
\end{aligned}
$$

the recursion relation

$$
P_{n}(x)=2 x P_{n-1}(x)+(n-1)(n-2) P_{n-2}(x),
$$

with $P_{0}(x)=1$. 
where in the second step we noted

$$
e^{z\left(\frac{1+u}{1-u}\right)}=e^{z} e^{2 z\left(\frac{u}{1-u}\right)} .
$$

Now the generating function of the ordinary Laguerre polynomials, $L_{m}(x)$, is[1]

$$
(1-u)^{-1} e^{-x\left(\frac{u}{1-u}\right)}=\sum_{m=0}^{\infty} L_{m}(x) u^{m},
$$

and it follows that

$$
C_{n}(z)=e^{z}\left[L_{n}(-2 z)-L_{n-1}(-2 z)\right]
$$

Since $L_{n}(x)-L_{n-1}(x)=-(x / n) L_{n-1}^{(1)}(x)$, where $L_{n-1}^{(1)}(x)$ is an associated Laguerre polynomial of degree $n-1$, which may be recast as a confluent hypergeometric function $n_{1} F_{1}(1-n, 2, x)$, we arrive at

$$
C_{n}(z)=(2 z){ }_{1} F_{1}(1-n, 2,-2 z) e^{z}, \quad n \geq 1,
$$

which is the desired result.

It is instructive to write $C_{n}(z) \equiv Q_{n}(z) e^{z}$, where $Q_{n}(z)$ is a polynomial of degree $n$ in $z$, so that

$$
c_{n}\left(t_{\phi}\right)=\left\langle\psi\left|Q_{n}\left(-t_{\phi} H\right)\right| \psi\left(-i t_{\phi}\right)\right\rangle,
$$

where $\left|\psi\left(-i t_{\phi}\right)\right\rangle$ is the wavepacket that has evolved for the complex time $-i t_{\phi}$ from the wavepacket $|\psi\rangle$ at $t=0$; since the point $t=-i t_{\phi}$ is further from the singularity at $2 i t_{0}$ than the point $t=0$, the influence of the singularity on $\left|\psi\left(-i t_{\phi}\right)\right\rangle$ is weaker than on $|\psi\rangle$. The point $t=-i t_{\phi}$, or, equivalently, $\tau=-i e^{i \phi}$, corresponds to the origin of the $u$-plane, i.e., the point about which a power series in $u$ is developed. Hence the conformal transformation permits the analytic continuation of $C(t)$ from a power series in $\tau$ to a power series in $u$ via a "connection" point $\tau=-i e^{i \phi}$ which lies within the circle of convergence of the power series in $\tau$, but on the side of this circle furthest from the singularity at $2 i t_{0}$.

We now explore some properties of the expansion coefficients $c_{n}\left(t_{\phi}\right)$. It is evident from Eqs. (47), (50), and (54) that since $H$ is Hermitian, and since $C_{n}(z)$ is a real function when $z$ is real,

$$
\left[c_{n}\left(t_{\phi}^{*}\right)\right]^{*}=c_{n}\left(t_{\phi}\right)
$$


Furthermore, since $t_{\phi}^{*}=t_{-\phi}$ we have

$$
c_{n}\left(t_{-\phi}\right)=\left[c_{n}\left(t_{\phi}\right)\right]^{*}
$$

We know from the previous section that $\left\langle\psi\left|e^{-t_{\phi} H}\right| \psi\right\rangle$ is analytic in the $t_{\phi}$-plane except on the negative imaginary axis, where $\phi= \pm \pi$. Hence $c_{0}\left(t_{\phi}\right)$ is analytic everywhere in the finite $t_{\phi}$-plane cut along the negative imaginary axis. Since $Q_{n}\left(-t_{\phi} H\right)$ is a polynomial, and therefore an analytic function of $t_{\phi} H$, we infer that $c_{n}\left(t_{\phi}\right)$ is also analytic everywhere in the finite cut $t_{\phi}$-plane, where $n$ is any nonnegative integer.

Although the expansion coefficients $c_{n}\left(t_{\phi}\right)$ are analytic throughout the range $|\phi|<\pi$, if we allow $\phi$ to move out of the range $|\phi|<\pi / 2$ the boundary line $\operatorname{Im} t / \operatorname{Re} t=\tan \phi$ crosses the cut that we have drawn along the positive imaginary $t$-axis. Hence if $\pi / 2 \leq|\phi|<\pi$ this cut is mapped into the unit circle and the expansion of $C(t)$ in powers of $u$, i.e., Eq. (46), no longer converges for all $u$ within the unit circle. Nevertheless, provided that $u$ is sufficiently small, i.e., provided that $t$ is sufficiently close to $-i t_{\phi}$, the expansion of $C(t)$ in powers of $u$ converges even for $\pi / 2 \leq|\phi|<\pi$. As $\phi$ increases from 0 to $\pi_{-}-$ where in general $a_{-}$and $a_{+}$, respectively, are numbers just below and just above the number $a-$ the point $-i t_{\phi}$ moves from the negative imaginary axis, into the right-half of the $t_{\phi}$-plane, and onto right edge of the positive imaginary axis. Thus, there is always a region in the right-half of the $t$-plane within which the expansion of $C(t)$ in powers of $u$ converges when $0 \leq \phi<\pi$; but as $\phi$ approaches $\pi_{-}$this region shrinks to the point $i$ on the cut in the $t$-plane. Similarly, as $\phi$ decreases from 0 to $-\pi_{-}$, the point $-i t_{\phi}$ moves from the negative imaginary axis, into the left-half of the $t_{\phi}$-plane, and onto left edge of the positive imaginary axis. Thus, there is always a region in the left-half of the $t$-plane within which the expansion of $C(t)$ in powers of $u$ converges when $-\pi<\phi \leq 0$; but as $\phi$ approaches $-\pi_{-}$this region again shrinks to the point $i$ on the cut. Recall that $\bar{C}(t)$ is the analytic continuation of $C(t)$ from the right-half to the left-half of the $t$-plane. As long as $|\phi|<\pi / 2$ we can express $\bar{C}(t)$ as a convergent power series in $u$ for all $u$ inside the unit circle; in analogy with Eq. (46) we have

$$
\bar{C}(t)=\sum_{n=0}^{\infty} \bar{c}_{n}\left(t_{\phi}\right) u^{n}
$$


where

$$
\bar{c}_{n}\left(t_{\phi}\right)=\left\langle\bar{\psi}\left|C_{n}\left(-t_{\phi} H\right)\right| \bar{\psi}\right\rangle .
$$

Recalling that $|\bar{\psi}\rangle=K|\psi\rangle$ and that $K$ is the antiunitary time-reversal operator, so that $K^{\dagger} K=1$ and $\left(\langle b| K^{\dagger}\right)|a\rangle=\left[\langle b|\left(K^{\dagger}|a\rangle\right)\right]^{*}$, we infer that for $|\phi|<\pi / 2$ we have

$$
\bar{c}_{n}\left(t_{\phi}\right)=c_{n}\left(t_{\phi}\right)
$$

This identity is hardly surprising since $C(t)$ and $\bar{C}(t)$ represent the same (multivalued) function of $t$, and as long as $|\phi|<\pi / 2$ there is a common region in the lower-half of the $t$-plane within which the expansions of both $C(t)$ and $\bar{C}(t)$ in powers of $u$ converge. However, once $\phi$ crosses the line $|\phi|=\pi / 2$ into the region $|\phi| \geq \pi / 2$ the characters of $c_{n}\left(t_{\phi}\right)$ and $\bar{c}_{n}\left(t_{\phi}\right)$ differ since there is no common region in the $t$-plane within which the expansions of $C(t)$ and $\bar{C}(t)$ both converge. On the cut along the negative imaginary axis in the $t_{\phi}$-plane, $c_{n}\left(t_{\pi}\right)$ and $\bar{c}_{n}\left(t_{-\pi}\right)$ are different branches of the same multivalued function of $t_{\phi}$.

An alternative form for $C_{n}(z)$, from which we may deduce the behavior of the coefficients $c_{n}\left(t_{\phi}\right)$ for large $n$, can be obtained using a standard expansion of the confluent hypergeometric function in terms of Bessel functions.[1] We find that

$$
C_{n}(z)=-2 \sum_{m=0}^{\infty} A_{m}(n) B_{m+1}(z), \quad n \geq 1
$$

where

$$
B_{m}(z)=(-z / 2 n)^{m / 2} J_{m}(\sqrt{-8 n z}),
$$

with $J_{n}(z)$ the regular Bessel function, and where $A_{0}(n)=1, A_{1}(n)=0$, $A_{2}(n)=1$, and

$$
A_{m+1}(n)=A_{m-1}(n)-[2 n /(m+1)] A_{m-2}(n), \quad m \geq 2 .
$$

For large $n$ and for $z$ fixed, real and negative (so that $\sqrt{-z}$ is real and positive) we have, using the asymptotic form of $J_{m}(x)$ for large $x$,

$$
B_{m}(z) \sim\left(\frac{-z^{2 m-1}}{2^{2 m+1} \pi^{2} n^{2 m+1}}\right)^{1 / 4} \cos \left(\sqrt{-8 n z}-\frac{1}{2} m \pi-\frac{1}{4} \pi\right), \quad n \sim \infty .
$$


It follows that for $n \sim \infty$, and $z$ fixed, real and negative, the first term in the series on the right side of Eq. (61) dominates, and we have

$$
C_{n}(z) \sim\left(\frac{-2 z}{\pi^{2} n^{3}}\right)^{1 / 4} \cos (\sqrt{-8 n z}+\pi / 4), \quad n \sim \infty .
$$

Of course, $-z$ is not a fixed number, but rather is the operator $t_{\phi} H$, which has a spectrum consisting of a discrete set of negative eigenvalues and a continuum of positive eigenvalues. From Eqs. (12) and (47) we have

$$
c_{n}\left(t_{\phi}\right)=c_{\mathrm{bd}, n}\left(t_{\phi}\right)+c_{\mathrm{cont}, n}\left(t_{\phi}\right),
$$

where, using Eq. (65),

$$
\begin{aligned}
& c_{\mathrm{bd}, n}\left(t_{\phi}\right) \\
& \equiv \sum_{m}\left|\psi_{\mathrm{bd}, m}\right|^{2} C_{n}\left(-t_{\phi} E_{\mathrm{bd}, m}\right) \\
& \sim\left(\frac{2}{\pi^{2} n^{3}}\right)^{1 / 4} \sum_{m}\left|\psi_{\mathrm{bd}, m}\right|^{2}\left(t_{\phi} E_{\mathrm{bd}, m}\right)^{1 / 4} \cos \left(\sqrt{8 n t_{\phi} E_{\mathrm{bd}, m}}+\pi / 4\right),
\end{aligned}
$$

and

$$
\begin{aligned}
& c_{\text {cont }, n}\left(t_{\phi}\right) \\
& \equiv \int d^{3} k|\psi(\mathbf{k})|^{2} C_{n}\left(-t_{\phi} E_{k}\right) \\
& \sim\left(\frac{2}{\pi^{2} n^{3}}\right)^{1 / 4} \int d^{3} k\left(t_{\phi} E_{k}\right)^{1 / 4}|\psi(\mathbf{k})|^{2} \cos \left(\sqrt{8 n t_{\phi} E_{k}}+\pi / 4\right) e^{-\eta k},
\end{aligned}
$$

where in the last step we inserted an unobtrusive factor of $e^{-\eta k}$ (with $\eta$ positive but infinitesimal) to ensure convergence at a later stage. We choose the phase of $E_{\mathrm{bd}, m}$ to be $\pi$, rather than $-\pi$, i.e., we write $E_{\mathrm{bd}, m}=e^{i \pi}\left|E_{\mathrm{bd}, m}\right|$, since the bound state poles are reached from the upper "physical" edge of the unitarity cut by following a path in the upper-half of the energy plane. Note that while $J_{m}(\sqrt{-8 n z})$ has a branch point singularity at $z=0$, due to the square root in the argument, $B_{m}(z)$ does not have a branch point at $z=0$ since $J_{m}(x)$ is proportional to $x^{m}$ for $x \sim 0$. On the other hand, if we take the liberty of using the asymptotic form of $B_{m}(z)$ - see Eq. (64) - for complex values of $z$, we infer that $B_{m}(z)$ is not single-valued when $\phi$ is varied, 
with $|z|$ held fixed, around a closed loop from $-\pi$ to $\pi$, and indeed we know that $c_{n}\left(t_{\phi}\right)$ is not single-valued when $\phi$ is varied from $-\pi$ to $\pi$.

Since $E_{\mathrm{bd}, m}<0$ the bound-state terms on the right side of Eq. (68) explode exponentially for $n \sim \infty$, unless $\phi= \pm \pi$. Hence $c_{\mathrm{bd}, n}\left(t_{\phi}\right)$ also explodes exponentially, unless $\phi= \pm \pi$ (excluding a potential with a Coulomb tail, a case considered below.) This singular behavior is related to the fact that $C_{\mathrm{bd}}(t)$ has an essential singularity at $t=\infty$, i.e., at $u=1$. As $t$ approaches $\infty$ in the lower-half $t$-plane, $C_{\mathrm{bd}}(t)$ explodes exponentially, and unless $\phi= \pm \pi$ there is a nonvanishing sector of the lower-half $t$-plane within which $t$ approaches $\infty$ and, concomitantly, $u$ approaches unity within the unit circle (this sector is the full half-plane if $\left.|\phi|=0_{+}\right)$. Therefore, unless $\phi= \pm \pi$, the power series in $u$ converges at $u=1$ only if we exclude from $|\psi\rangle$ the bound-state eigenvectors of $H$. Thus we replace $|\psi\rangle$ by $P_{\text {cont }}|\psi\rangle$ where $P_{\text {cont }}=1-P_{\mathrm{bd}}$ and where $P_{\text {cont }}$ and $P_{\mathrm{bd}}$ are projection operators with $P_{\mathrm{bd}}$ defined as

$$
P_{\mathrm{bd}}=\sum_{m}\left|\chi_{\mathrm{bd}, m}\right\rangle\left\langle\chi_{\mathrm{bd}, m}\right|
$$

where, if the potential is short-range (i.e., no Coulomb tail), the sum is over all bound states. The omission of these bound states does not affect the rate for a continuum process (if the potential is short-range), but it may affect the energy shift of the system. Note that while the compound states also give rise to an essential singularity, they need not be omitted; the sector of the $t$-plane in which they explode corresponds to letting $u$ approach unity from without the unit circle, provided that $|\phi|<\Xi_{0}$.

To obtain the large- $n$ behavior of $c_{\text {cont }, n}\left(t_{\phi}\right)$, we change variables from $\mathbf{k}$ to $\mathbf{k} / \sqrt{n}$ on the right side of Eq. (70). Let us first treat the case of a short-range potential, and for simplicity let us assume that the angular momentum quantum number $l$ is zero. We can factor $|\psi(\mathbf{k} / \sqrt{n})|^{2}$ out of the integral as $|\psi(\mathbf{0})|^{2}$, and we find that

$$
c_{\text {cont }, n}\left(t_{\phi}\right) \sim \frac{15 \pi}{2^{9 / 2}}\left(\frac{\mu}{t_{\phi}}\right)^{3 / 2} \frac{|\psi(\mathbf{0})|^{2}}{n^{5 / 2}}, \quad n \sim \infty
$$

where we used

$$
\int d^{3} k\left(t_{\phi} E_{k}\right)^{1 / 4} \cos \left(\sqrt{8 t_{\phi} E_{k}}+\pi / 4\right) e^{-\eta k}=\frac{15 \pi^{3 / 2}}{2^{19 / 4}}\left(\frac{\mu}{t_{\phi}}\right)^{3 / 2} .
$$


We pause for a few remarks. First, while the integral on the left side of Eq. (73) is formally defined only for $t_{\phi}$ real and positive, both sides of Eq. (72) are analytic everywhere in the finite $t_{\phi}$-plane cut along the negative real axis. Second, if $l \neq 0$ we must modify Eq. (72) by including a factor proportional to $1 / n^{2 l}$. Finally, note that the large- $n$ behavior of $c_{\text {cont }, n}\left(t_{\phi}\right)$ is determined by small $k$, i.e., by the continuum eigenvalues of $H$ close to threshold. Since the large- $t$ behavior of $C_{\text {cont }}(t)$ is also determined by the continuum eigenvalues close to threshold, we infer that the large- $t$ behavior of $C_{\text {cont }}(t)$ is determined by those terms with large $n$ in the power series on the right side of Eq. (46).

It follows that if the potential is short-range, $c_{\text {cont }, n}\left(t_{\phi}\right)$ decreases as $n^{-(2 l+5) / 2}$ as $n$ increases. We now consider the case where the potential has an attractive Coulomb tail. We must first reconsider $c_{\mathrm{bd}, n}\left(t_{\phi}\right)$. There are an infinite number of Rydberg bound states converging to threshold for which $\left|8 n t_{\phi} E_{\mathrm{bd}, m}\right|<1$ no matter how large is $n$. Such states do not yield divergent terms on the right side of Eq. (68) and, as noted earlier, cannot be distinguished from continuum states converging to threshold; therefore, they should not be projected out of the wavepacket when $\phi \neq \pm \pi$. We write $E_{\mathrm{bd}, m}=-Z^{2} e^{2} /\left[2\left(m^{*}\right)^{2} a_{0}\right]$, where $m^{*}=m-\delta$, with $\delta$ the quantum defect, which is roughly independent of $m$ for $m \gg 1$. We must retain at least those bound states for which $m^{*}$ is roughly greater than or of the order of $\sqrt{n}$. If $m * \gg 1$ we can relate $\left|\psi_{\mathrm{bd}, m}\right|^{2}$ to $|\psi(\mathbf{k})|^{2}$ as follows. The wavepacket $|\psi\rangle$ contains Rydberg bound states with population $\left|\psi_{\mathrm{bd}, m}\right|^{2} d m^{*}$ in the interval $\left(m^{*}, m^{*}+d m^{*}\right)$, and continuum states with population $|\psi(\mathbf{k})|^{2} d^{3} k$ in the interval $d^{3} k$ centered at $\mathbf{k}$. Just above threshold, where $k a_{0} \ll Z$, we can replace $|\psi(\mathbf{k})|^{2}$ by $2 \pi \gamma|\tilde{\psi}(\mathbf{0})|^{2}$, where we used Eq. (19) noting that $e^{-2 \pi \gamma} \ll 1$. Since $d E_{\mathrm{bd}, m} / d m^{*}=Z^{2} e^{2} /\left[\left(m^{*}\right)^{3} a_{0}\right]$ and $d E_{k} / d k=k / \mu$, and since the differential populations with respect to energy must be the same for bound states just below, and continuum states just above, threshold, we have, writing $d^{3} k=4 \pi k^{2} d k$,

$$
\left|\psi_{\mathrm{bd}, m}\right|^{2}=\left(\frac{8 \pi^{2} Z^{3}}{\left(m^{*} a_{0}\right)^{3}}\right)|\tilde{\psi}(\mathbf{0})|^{2}
$$

It follows from Eqs. (68) and (74) that, with $t_{\phi}$ on the upper edge of the cut along the negative real axis of the $t_{\phi}$-plane, and with $E_{\mathrm{bd}, m}=$ 
$e^{i \pi}\left|E_{\mathrm{bd}, m}\right|$

$$
\begin{aligned}
c_{\mathrm{bd}, n}\left(t_{\phi}\right) \sim 8 \pi^{2} Z^{3}|\tilde{\psi}(0)|^{2} & \left(\frac{2}{\pi^{2} n^{3}}\right)^{1 / 4} \\
& \cdot \sum_{m=1}^{\infty} \frac{\left(t_{\phi} E_{\mathrm{bd}, m}\right)^{1 / 4}}{\left(m^{*} a_{0}\right)^{3}} \cos \left(\sqrt{8 n t_{\phi} E_{\mathrm{bd}, m}}+\pi / 4\right),
\end{aligned}
$$

where we have extended the lower limit of the sum to 1 since the leading $n$-behavior of the sum is relatively insensitive to the lower limit. In Appendix $\mathrm{D}$ we analyse the large- $n$ behavior $c_{\mathrm{bd}, n}\left(t_{\phi}\right)$ and $c_{\mathrm{cont}, n}\left(t_{\phi}\right)$ for all complex $t_{\phi}$. To summarise the results of Appendix $\mathrm{D}$, when the potential has an attractive Coulomb tail we find that

$$
c_{n}\left(t_{\phi}\right) \sim \frac{i}{n^{2}}\left(\frac{3 \pi^{2} Z \mu|\tilde{\psi}(\mathbf{0})|^{2}}{2^{5 / 2} a_{0} t_{\phi}}\right), \quad-\pi<\phi<\pi,
$$

for $t_{\phi}$ on the first sheet, and

$$
c_{n}\left(t_{\phi}\right) \sim-\frac{i}{n^{2}}\left(\frac{3 \pi^{2} Z \mu|\tilde{\psi}(\mathbf{0})|^{2}}{2^{5 / 2} a_{0} t_{\phi}}\right), \quad \pi<\phi<3 \pi,
$$

for $t_{\phi}$ on the second sheet. Hence, $c_{n}\left(t_{\phi}\right)$ has not only a branch cut on the negative real $t_{\phi}$-axis, but also a discontinuity which amounts to a sign reversal.

\section{Green function}

The inclusive rate at which a continuous stationary or quasistationary process occurs if $\mathcal{E}$ is the positive energy of the system is $-2 \operatorname{Im} R(\mathcal{E}+i \eta)$ where $\eta$ is positive but infinitesimal and where $R(E)$ is a Green function matrix element of the form

$$
R(E) \equiv\langle\psi|G(E)| \psi\rangle .
$$

The real part of $R(\mathcal{E}+i \eta)$ includes the energy shift of the system. For example, if the system consists of an infinitely heavy particle (at rest) and a light particle that is incident from infinity in the unperturbed state represented by $\left|\Psi_{0}\right\rangle$, we obtain the rate for the light particle to 
scatter by putting $|\psi\rangle=W\left|\Psi_{0}\right\rangle$ where $W$ is the interparticle potential. As another example, consider a system that consists of an atom, initially bound in the unperturbed state represented by $\left|\Psi_{0}\right\rangle$; if this atom is exposed to weak monochromatic radiation, we obtain the rate for the atom to decay by putting $|\psi\rangle=V_{+}\left|\Psi_{0}\right\rangle$ where $V_{+}$is the one-photon absorption operator.

Provided that $E$ lies in the upper half of the complex $E$-plane we can represent $R(E)$ by the integral

$$
\begin{aligned}
R(E) & =-i \int_{0}^{\infty} d t e^{i E t} C(t) \\
& =-i t_{0} \int_{0}^{\infty} d \tau e^{i\left(t_{0} E\right) \tau} C(t) .
\end{aligned}
$$

Since $C(t)$ is bounded throughout the sector $0 \leq \arg (\tau)<\Xi_{0}$ we can rotate the contour of integration into this sector and extend the integral representation to a sector in the lower-right quadrant of the $E$-plane. To analytically continue $R(E)$ into a sector of the $E$-plane that includes the negative real $E$-axis we first recall that both $C_{\text {res }}(t)$ and $C_{\mathrm{bg}}(t)$ are bounded throughout the lower right quadrant of the $\tau$-plane, but $C_{\mathrm{bd}}(t)$ is unbounded in this quadrant. Therefore, provided that we project out the bound states, and choose $E$ to lie temporarily on the left edge of the positive imaginary $E$-axis, we can rotate the integration contour into the lower-right quadrant of the $\tau$-plane so that it runs along the right edge of the negative imaginary $\tau$-axis. Subsequently, we can move $E$ from the upper- to the lower-left quadrant of the $E$-plane. Although we subtracted the bound-state contribution to $R(E)$, this contribution is analytic, having only poles on the negative real $E$-axis. Hence, we have analytically continued $R(E)$ from the upper- to the lower-left quadrant of the $E$-plane.

To deal with all values of $E$ in the lower half of the complex $E$-plane we introduce $\bar{R}(E) \equiv\langle\bar{\psi}|G(E)| \bar{\psi}\rangle$, where we recall that $|\bar{\psi}\rangle=K|\psi\rangle$. For $\operatorname{Im} E<0$ we can represent $\bar{R}(E)$ by the integral

$$
\bar{R}(E)=-i t_{0} \int_{0}^{-\infty} d \tau e^{i\left(t_{0} E\right) \tau} \bar{C}(t) .
$$

Since $\bar{C}(t)$ is bounded throughout the sector $\pi-\Xi_{0} \leq \arg (\tau)<\pi$ we can rotate the contour of integration into this sector, and again extend 
the validity of the integral representation, now to a sector in the upperright quadrant of the $E$-plane. To analytically continue $\bar{R}(E)$ into the upper-left quadrant of the $E$-plane we proceed as for $R(E)$; we project out the bound states, choose $E$ to lie temporarily on the left edge of the negative imaginary $E$-axis, rotate the integration contour through the lower left quadrant of the $\tau$-plane, so that it runs along the left edge of the negative imaginary $\tau$-axis, and subsequently move $E$ into the upper-left quadrant.

Since $C(t)$ and $\bar{C}(t)$ are continuous in the lower-half $t$-plane, the integral representations of $R(E)$ and $\bar{R}(E)$ are identical when both contours are chosen to be the negative imaginary $\tau$-axis. Hence $R(E)$ and $\bar{R}(E)$ are identical in the lower-left quadrant of the $E$-plane; it follows that $\bar{R}(E)$ is the analytic continuation of $R(E)$ from the upper part of the $E$-plane to the lower part, on the first sheet of the Riemann energy surface cut from 0 to $\infty$ along a line parallel to the integration contour of either $R(E)$ or $\bar{R}(E)$. At the cut, $R(E)$ and $\bar{R}(E)$ are different branches of the same function. As noted above, the integration contours of $R(E)$ and $\bar{R}(E)$, respectively, can be rotated into sectors of the lower- and upper-right quadrants of the $E$-plane; hence the cut can be rotated into these sectors, thereby extending the region of the Riemann energy surface covered by the integral representations. However, these integral representations do not permit the entire Riemann surface to be covered. As we see below, the entire surface can be covered by the series representation. The wider region of analyticity afforded by the series representation is a reward for performing the integration over time.

Using the expansion of $C(t)$ in powers of $u$ we have

$$
R(E)=-i t_{0} \int_{0}^{\infty} d \tau \sum_{n=0}^{\infty} c_{n}\left(t_{\phi}\right) u^{n} e^{i\left(E t_{0}\right) \tau} .
$$

Rotating the integration contour to the line $\arg (\tau)=\phi$, interchanging sum and integral, and recalling that

$$
\mathcal{I}_{n}(a) \equiv \int_{0}^{\infty} d \tau e^{i a \tau}\left(\frac{\tau+i}{\tau-i}\right)^{n}
$$

we arrive at the series representation

$$
R(E)=-i t_{\phi} \sum_{n=0}^{\infty} c_{n}\left(t_{\phi}\right) \mathcal{I}_{n}\left(E t_{\phi}\right),
$$


where for the moment $|\phi|<\pi / 2$. We obtain the same series representation of $\bar{R}(E)$ after expanding $\bar{C}(t)$ in powers of $u$, and rotating the integration contour to the line $\arg (\tau)=\pi+\phi$. The resolvent itself has the series representation

$$
\begin{aligned}
P_{\text {cont }} G(E) & =-i t_{0} P_{\text {cont }} \int_{0}^{\infty} d \tau e^{i\left(E t_{0}\right) \tau} U(t) \\
& =-i t_{\phi} \sum_{n=0}^{\infty} \mathcal{I}_{n}\left(E t_{\phi}\right) P_{\text {cont }} C_{n}\left(-t_{\phi} H\right),
\end{aligned}
$$

as discussed in the Introduction. Since $R(E)$ is defined by an integral whose contour is the positive real $\tau$-axis, this contour must be mapped into the unit circle, as it is if $\phi$ lies in the range $0<\phi<\pi / 2$; hence the series representation of $R(E)$ is defined for $\phi$ in this range. On the other hand, $\bar{R}(E)$ is defined by an integral whose contour is the negative real $\tau$-axis, which is mapped into the unit circle if $\phi$ lies in the range $-\pi / 2<\phi<0$; hence the series representation of $\bar{R}(E)$ is defined for $\phi$ in this range. It follows that when $E$ is real and positive, the physical branch is specified by $0<\phi<\pi / 2$, whereas the unphysical branch is specified by $-\pi / 2<\phi<0$.

We now prove the useful result that we can pass (discontinuously) from one branch of $R(E)$ to the other branch, i.e., to $\bar{R}(E)$, by simply changing the sign of the phase $\phi$. We first observe that, according to Eq. (43), changing the sign of $\phi$ and complex conjugating $u$ amounts to changing $\tau$ into $-\tau^{*}$, or, equivalently, $t$ into $-t^{*}$. It follows from Eqs. (46) and (57) that complex conjugating $C(t)$ and changing the sign of $\phi$ yields $C\left(-t^{*}\right)$, which, using Eq. (36), is the same as $[\bar{C}(t)]^{*}$. Hence, simply changing the sign of $\phi$, without complex conjugation, turns $C(t)$ into $\bar{C}(t)$. Furthermore, changing the sign of $\phi$, from positive to negative, say, transforms the contour of integration for $R(E)$ discontinuously into the contour for $\bar{R}(E)$; if we vary $\phi$ continuously from a positive to a negative value the integration contour - the broken line in Fig. 3 - starts as a path in the upper semicircle, it moves upwards towards to the boundary of this semicircle, and upon reaching this boundary jumps discontinuously to the boundary of the lower semicircle, and from there to a path inside the lower semicircle. Thus we have arrived at the result we set out to prove.

An important task that remains is the analytic continuation of the series representation of $R(E)$ from $|\phi|<\pi / 2$ to $|\phi|>\pi / 2$. To carry 


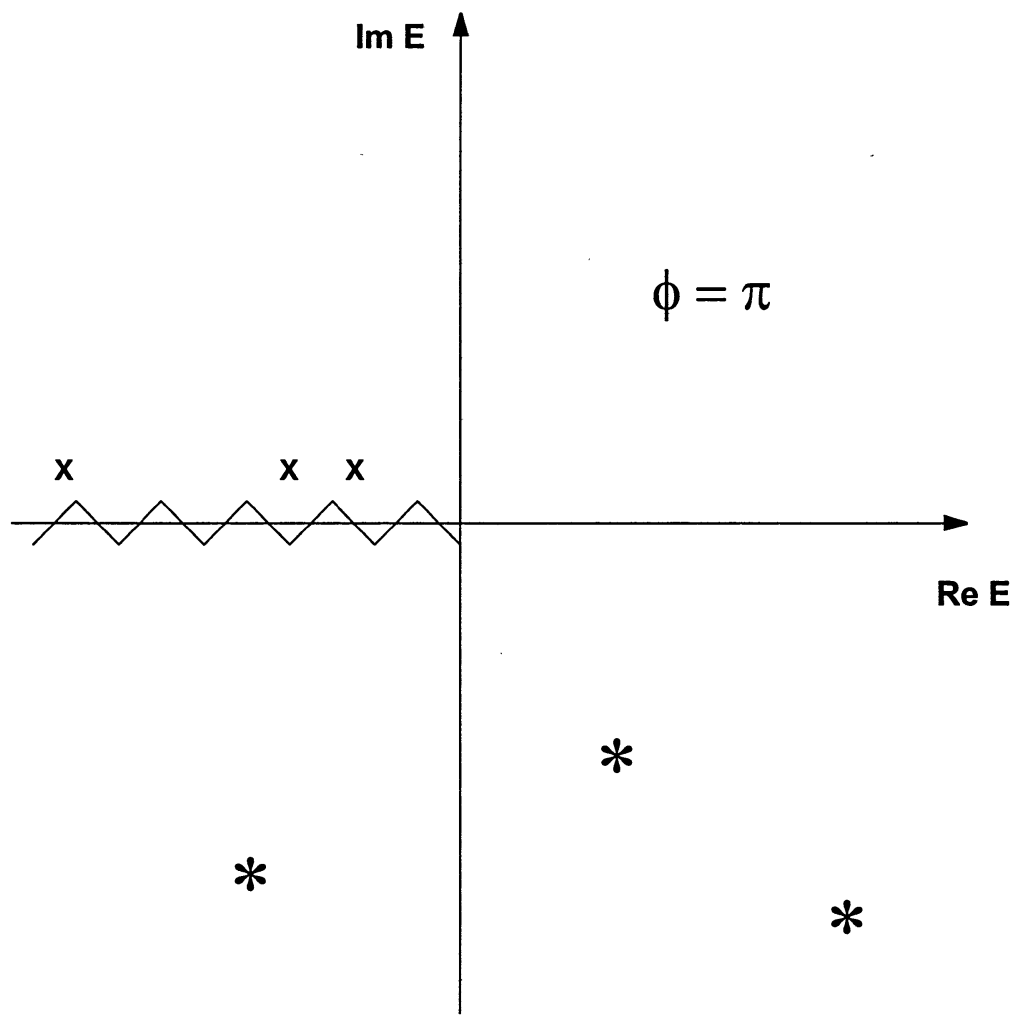

Figure 4: The physical sheet of the Riemann energy surface cut along the negative real axis. Bound state poles $(x)$ lie on the upper edge of the cut and resonance poles $(\star)$ lie in the lower-half plane. The series representation of the resolvent applies on this sheet if we choose the phase parameter to be $\phi=\pi$. 
out this task we must analyse the integrals $\mathcal{I}_{n}(a)$, defined by Eq. (83). These integrals are not formally defined when $\operatorname{Im} a \leq 0$ since the integrands do not vanish for $\tau \sim \infty$. We can analytically continue $\mathcal{I}_{n}(a)$ to the region $\operatorname{Im} a \leq 0$ by rotating the integration contour into the upperhalf $\tau$-plane. However, if $n \geq 1$ the integrand is singular along the positive imaginary $\tau$-axis, and therefore the (positive) angle through which we rotate the contour must be less than $\pi / 2$, which only permits analytic continuation of $\mathcal{I}_{n}(a)$ to the lower-right quadrant of the complex $a$-plane. To analytically $\mathcal{I}_{n}(a)$ continue to the lower-left quadrant of the $a$-plane we move $a$ temporarily to the upper edge of the negative real axis in the $a$-plane, rotate the integration contour through the angle $-\pi$, so that it runs along the lower edge of the negative imaginary $\tau$-axis, and subsequently move $a$ into the lower-half of the $a$-plane. However, when $n \geq 1, \mathcal{I}_{n}(a)$ has a branch cut extending from 0 to $\infty$ in the $a$-plane since we cannot move $a$ continuously around a closed loop by continuously rotating the contour of the integral representation of $\mathcal{I}_{n}(a)$. The direction of this cut is a matter of convention, and that convention has already been established. Inspection of Eq. (86) reveals that the choice of the cut for $\mathcal{I}_{n}(a)$ determines the cut in the $E$-plane for the series representation of $R(E)$, and we want that to be the same as for the integral representation of $R(E)$. Thus we draw the cut for $\mathcal{I}_{n}(a)$ along the positive real axis in the $a$-plane, which implies, according to Eq. (86), that the series representation of $R(E)$ has a cut in the $E$-plane along the line $\arg E=-\phi$. This conforms to our specification that $R(E)$ and $\bar{R}(E)$, respectively, are analytic in the upper and lower halves of the $E$-plane, and have integral representations defined in the ranges $0<\phi<\pi / 2$ and $-\pi / 2<\phi<0$. We now see how the series can represent $R(E)$ over the entire Riemann energy surface. If we let $\phi$ increase from 0 to $\pi_{-}$, the branch cut swings from the positive real $E$-axis, down past the resonance poles in the lower-half E-plane, to the lower edge of the negative real $E$-axis. Hence, fixing $\phi$ to be $\pi_{-}$, we see that $R(E)$ is defined over an entire energy plane, and its poles are the bound-state poles on the upper edge of the cut and the resonance poles in the lower half of the energy-plane. See Fig. 4. This energy plane is the physical sheet of the Riemann surface, since $R(E)$ is the branch corresponding to outgoing-wave boundary conditions when $E$ is real and positive. Similarly, if we let $\phi$ derease from 0 to $-\pi_{-}$, the branch cut swings up past the resonance poles in the upper-half $E$-plane, to the upper edge of the negative real $E$-axis. Hence, fixing $\phi$ to be $-\pi_{-}$, we see that $\bar{R}(E)$ is also defined over an entire energy plane, and it has 




Figure 5: The unphysical sheet of the Riemann energy surface cut along the negative real axis. Bound state poles $(x)$ lie on the lower edge of the cut and resonance poles $(\star)$ lie in the upper-half plane. The series representation of the resolvent applies on this sheet if we choose the phase parameter to be $\phi=-\pi$.

bound-state poles on the lower edge of the cut; but its resonance poles are conjugate to those of $R(E)$, as shown in Fig. 5, and the energy plane is the unphysical sheet of the Riemann surface since $\bar{R}(E)$ is the branch corresponding to ingoing-wave boundary conditions when $E$ is real and positive.

To determine the rate of convergence of the series representation of $R(E)$ we need to analyse the behavior of $\mathcal{I}_{n}(a)$ for large $n$. The integrand on the right side of Eq. (83) oscillates rapidly when either $n$ or $a$ is large. To treat large $n$ it is useful to change to the variable 
$\theta=\tan ^{-1}(\tau)$. We have

$$
\mathcal{I}_{n}(a)=(-1)^{n} \int_{0}^{\pi / 2} d \theta \sec ^{2} \theta e^{i a \tan \theta-2 i n \theta} .
$$

We start by assuming that $a$ is real and positive and that $n>a / 2$. There is a point of stationary phase at $\theta=\theta_{0}$, where

$$
\cos ^{2} \theta_{0}=a /(2 n),
$$

and if $n \gg a / 2$ we have, using the method of stationary phase,

$$
\mathcal{I}_{n}(a) \sim(-1)^{n} \sec ^{2} \theta_{0}\left(\frac{2 \pi}{n\left|s^{\prime \prime}\left(\theta_{0}\right)\right|}\right)^{1 / 2} e^{i n s\left(\theta_{0}\right)+i \pi / 4},
$$

where $s^{\prime \prime}(\theta)$ is the second derivative with respect to $\theta$ of

$$
s(\theta)=(a / n) \tan \theta-2 \theta .
$$

Noting that $s^{\prime \prime}\left(\theta_{0}\right)=4 \tan \theta_{0}$ and that

$$
\tan \theta_{0} \approx(2 n / a)^{1 / 2}(1-a / 4 n)
$$

we find that

$$
\mathcal{I}_{n}(a) \sim\left(\frac{2 \pi^{2} n}{a^{3}}\right)^{1 / 4} e^{i \sqrt{8 a n}+i \pi / 4}, \quad a>0 .
$$

Evidently, when $a$ is real and positive $\mathcal{I}_{n}(a)$ grows as $n^{1 / 4}$ with increasing $n$. When $a$ is real and negative, $\operatorname{Re} \mathcal{I}_{n}(a)=0$; this follows upon rotating the contour of integration on the right-side of Eq. (83) from the real positive axis to the negative imaginary axis. For complex values of $a$, in the plane cut along the positive real axis, the point of stationary phase becomes a saddle point, and the expression on the right-side of Eq. (91), when analytically continued, becomes exponentially small, i.e., it decreases exponentially with increasing $n$ as $e^{-\operatorname{Im} \sqrt{8 a n}}$. However, we must include not just the contribution from the saddle point, but also the "surface" contribution, at the lower limit of the integral $\mathcal{I}_{n}(a)$; integration by parts yields the following asymptotic expansion in powers of $1 / n$, applicable as long as the saddle-point contribution is negligible:

$$
\mathcal{I}_{n}(a) \sim \frac{(-1)^{n+1} i}{n} \sum_{j=0}^{\infty} \frac{f_{j}(a)}{n^{j}}
$$


where $f_{j}(a)$ is a polynomial of degree $j$ with real coefficients. For example, $f_{0}(a)=1 / 2, f_{1}(a)=a / 4$, and $f_{2}(a)=\left(a^{2}-2\right) / 8$.

Suppose that $E$ is real and positive. Let us set $\phi=0_{ \pm}$in the series representation of $R(E)$. The large- $n$ behavior of the factor $\mathcal{I}_{n}\left(E t_{\phi}\right)$ is given by Eq. (91). These factors, while oscillating as $n$ increases, also weakly magnify each term of the series for $R(E)$. However, since the $c_{n}\left(t_{\phi}\right)$ decrease more rapidly than the $\mathcal{I}_{n}\left(E t_{\phi}\right)$ increase, the series converges, albeit logarithmically. We are now at the stage where we can work through some examples which support the validity of the series representations of $G(E)$ and $R(E)$, i.e., Eqs. (86) and (84). We do this in Appendix B.

If $\phi$ is chosen to be in the range $0<|\phi|<\pi$, the saddle-point contribution to $\mathcal{I}_{n}\left(t_{\phi} E\right)$ can be neglected for $n$ sufficiently large, i.e., for $\sqrt{8 n t_{0} E} \sin (|\phi / 2|) \gg 1$. In this case we can use the asymptotic expansion of Eq. (92) to express, in a rather useful form, the contribution of the higher terms to the series representation of $G(E)$. Combining Eqs. (86), (50), and (54) gives

$$
P_{\text {cont }} G(E)=P_{\text {cont }} e^{-t_{\phi} H}\left(\frac{1}{E}+i t_{\phi}\left(2 t_{\phi} H\right) S(E, \phi)\right),
$$

where $S(E, \phi)$ is the infinite sum

$$
S(E, \phi)=\sum_{n=1}^{\infty} \mathcal{I}_{n}\left(t_{\phi} E\right){ }_{1} F_{1}\left(1-n, 2,-2 t_{\phi} H\right) .
$$

Using Eqs. (92) and (94), and neglecting the contribution from the saddle point, gives, after interchanging the order of the sums,

$$
S(E, \phi) \sim-i \sum_{j=1}^{\infty} f_{j-1}\left(t_{\phi} E\right) \sum_{n=1}^{\infty} \frac{(-1)^{n}}{n^{j}}{ }_{1} F_{1}\left(1-n, 2,-2 t_{\phi} H\right) .
$$

We now use the Watson transform:

$$
\sum_{n=1}^{\infty}(-1)^{n} F(n)=\frac{1}{2 i} \int_{\Gamma} d n \frac{F(n)}{\sin (n \pi)}
$$

where $\Gamma$ is a counterclockwise contour which encloses the section of the real positive $n$-axis extending from a point in the interval $(0,1)$ to 
infinity. We distort $\Gamma$ into a line, $\Gamma^{\prime}$, parallel to the imaginary $n$-axis, with $0<\operatorname{Re} n<1$, a region where it is permissible to use the integral representation

$$
{ }_{1} F_{1}(1-n, 2, z)=\frac{\sin (n \pi)}{n \pi} \int_{0}^{1} d u e^{z u}\left(\frac{1-u}{u}\right)^{n} .
$$

It follows that

$$
S(E, \phi) \sim-\sum_{j=1}^{\infty} \frac{f_{j-1}\left(t_{\phi} E\right)}{2 \pi} \int_{\Gamma} d n \frac{1}{n^{j+1}} \int_{0}^{1} d u e^{-2 t_{\phi} H u}\left(\frac{1-u}{u}\right)^{n} .
$$

Define $\omega \equiv \ln [(1-u) / u]$. If $u<1 / 2$ we have $\omega>0$, while if $u>1 / 2$ we have $\omega<0$. Now, if $u>1 / 2$ we can bend the contour $\Gamma^{\prime}$ back into $\Gamma$; we obtain zero since $\Gamma$ no longer encloses any poles. If $u<1 / 2$ we can bend $\Gamma^{\prime}$ into a contour $\Gamma^{\prime \prime}$ that encloses the whole of the negative real $n$-axis and, in addition, a section $0 \leq n \leq n_{0}<1$ of the positive real $n$-axis. We use, integrating by parts in the first step,

$$
\begin{aligned}
\int_{\Gamma^{\prime \prime}} d n \frac{e^{\omega n}}{n^{j+1}} & =\frac{\omega^{j}}{j !} \int_{\Gamma^{\prime \prime}} d n \frac{e^{\omega n}}{n} \\
& =\frac{\omega^{j}}{j !}\left(\int_{-\infty}^{n_{0}+i \eta} d n \frac{e^{\omega n}}{n}+\int_{n_{0}-i \eta}^{-\infty} d n \frac{e^{\omega n}}{n}\right)
\end{aligned}
$$

where $\eta$ is positive but infinitesimal. Transforming the integration variable from $n$ to $-n / \omega$, and noting that the exponential integral, $E_{1}(z)$, has a logarithmic branch point at $z=0$, with a branch cut customarily drawn along the negative real $z$-axis, we obtain

$$
\begin{aligned}
\int_{\Gamma^{\prime \prime}} d n \frac{e^{\omega n}}{n} & =E_{1}\left(-\omega n_{0}+i \eta\right)-E_{1}\left(-\omega n_{0}-i \eta\right) \\
& =-2 i \pi .
\end{aligned}
$$

Putting all this together, we obtain

$$
S(E, \phi) \sim i \sum_{j=1}^{\infty} \frac{f_{j-1}\left(t_{\phi} E\right)}{j !} \int_{0}^{1 / 2} d u e^{-2 t_{\phi} H u}\left[\ln \left(\frac{1-u}{u}\right)\right]^{j} .
$$

The evolution operator $e^{-2 t_{\phi} H u}$ acts for at most one unit of time, and can be calculated rapidly (using a Padé approximant — see Appendix 
C). The first few terms - say the first $N$ - on the right side of Eq. (94) should be evaluated exactly, and accordingly the first $N$ terms built from the right side of Eq. (92) should be subtracted from $S(E, \phi)$, with $N$ chosen so that for $n>N$ the following conditions are satisfied: (i) The asymptotic expansion of Eq. (92) converges when only a small number of terms are included, and (ii) the saddle-point contribution to $\mathcal{I}_{n}\left(t_{\phi} E\right)$ can be neglected.

\section{Conclusion}

We have derived a series representation of the resolvent $G(E)$ which incorporates a complex unit of time, $t_{0} e^{i \phi}$, whose phase $\phi$ specifies the branch. This permits a real basis to be used to construct a matrix representation of the Hamiltonian. The foundation of our approach is the analytic continuation of the temporal correlation function in the complex time-plane. Using analytic continuation we can extrapolate the correlation function from a relatively small interval, of duration $t_{0}$, to an interval of asymptotically large duration. Since much of the dynamics take place during a time that, typically, is comparable to $t_{0}$, it should be possible to employ a basis efficiently; it is unnecessary to span spatial regions that are reached by the particle(s) at times very much larger than $t_{0}$, where the wavefunction takes on its asymptotic form.

This work was supported by the NSF, Grant No. PHY-9722048.

\section{Appendix A: Equivalence of Two Forms of the Integral $\mathcal{I}_{n}(a)$}

In this appendix we show that Eqs. (4) and (8) are equivalent definitions of $\mathcal{I}_{n}(a)$. To do this we use a standard contour integral representation of the Laguerre polynomial:

$$
L_{n-1}^{(1)}(x)=\frac{1}{2 \pi i} \oint e^{-x \zeta} d \zeta\left(\frac{1+\zeta}{\zeta}\right)^{n},
$$


where the contour runs counterclockwise and encloses the point $\zeta=0$. Substituting this representation into the right side of Eq. (8) gives

$$
\mathcal{I}_{n}\left(E t_{\phi}\right)=-\frac{1}{\pi} \oint d \zeta\left(\frac{1+\zeta}{\zeta}\right)^{n} \int_{0}^{\infty} d H e^{-t_{\phi} H(1+2 \zeta)}\left(\frac{1}{E-H}-\frac{e^{-t_{\phi} H}}{E}\right) .
$$

The integrals of Eq. (105) are defined as long as (i) $E$ is not real and positive and (ii) $\operatorname{Re} t_{\phi}(1+2 \zeta)>0$. This second condition implies that the closed contour excludes the point $\zeta=-1 / 2$. The first condition can be dropped after integrating over $H$. Performing the integration over $H$ gives, with $a=E t_{\phi}$,

$$
\mathcal{I}_{n}(a)=\frac{1}{2 \pi} \oint d \zeta\left(\frac{1+\zeta}{\zeta}\right)^{n}\left(e^{-a(1+2 \zeta)} E_{1}[-a(1+2 \zeta)]+\frac{1}{2 a(1+\zeta)}\right)
$$

where the branch cut of $E_{1}(z)$, the exponential integral, is along the negative real $z$-axis; the contour of integration in Eq. (106) excludes the branch point at $\zeta=-1 / 2$ and is drawn to exclude the cut along the line $\arg a(1+2 \zeta)=0$. Changing variables from $\zeta$ to $\tau=1+2 \zeta$ we obtain

$$
\mathcal{I}_{n}(a)=\frac{1}{2 \pi} \oint d \tau\left(\frac{\tau+1}{\tau-1}\right)^{n}\left(e^{-a \tau} E_{1}(-a \tau)+\frac{1}{a(\tau+1)}\right),
$$

where the new (still counterclockwise) contour excludes both the point $\tau=0$ and the branch cut along the line arg $a \tau=0$, but includes the point $\tau=1$. We now deform this contour so that it wraps around the branch cut and runs along the circle at infinity. Since $e^{z} E_{1}(z) \sim$ $(1 / z)-\left(1 / z^{2}\right)$ for $z \sim \infty$ the integral over the circle at infinity vanishes. The integral around the branch cut can be simplified after noting that as $z$ moves from the upper to the lower edge of the negative real $z$-axis, $E_{1}(z)$ increases by $2 \pi i$. It follows that

$$
\mathcal{I}_{n}(a)=i \int_{0}^{-\infty} d \tau e^{-a \tau}\left(\frac{\tau+1}{\tau-1}\right)^{n}
$$

By rotating the integration contour through $90^{\circ}$, from the negative real $\tau$-axis to to the negative imaginary $\tau$-axis, we reproduce Eq. (4). 


\section{Appendix B: Analytical Tests}

In this appendix we give two examples where we use the series representations given by Eqs. (86) and (84) to establish some known formal results. In our first example we use Eq. (86) to derive the standard expression $1 /(E-H)$ for $G(E)$ when the potential is arbitrary; but we assume that the part of $G(E)$ which accounts for propagation far off the energy shell is relatively unimportant. We choose both $t_{0}$ and $\phi$ to be small, such that $E t_{0}$ is significantly smaller than 1 and $|\phi| \ll \pi$. Hence the exponential $e^{i \sqrt{8 t_{\phi} E n}}$ is almost undamped and is slowly oscillating. Therefore a large number of terms contribute to the series on the right side of Eq. (86) and the sum is determined primarily by large- $n$ terms. Consequently, we can substitute the asymptotic forms for $C_{n}\left(-t_{\phi} H\right)$ and $\mathcal{I}_{n}\left(E t_{\phi}\right)$ - see Eqs. (65) and (91) - into the series of Eq. (86) to give

$$
\begin{aligned}
G(E) \approx-i t_{\phi}\left(\frac{H}{E}\right)^{1 / 4} \sum_{n \gg 1}^{\infty} & \left(\frac{2}{t_{\phi} E n}\right)^{1 / 2} \\
\cdot & e^{i \sqrt{8 t_{\phi} E n}+i \pi / 4} \cos \left(\sqrt{8 n t_{\phi} H}+\pi / 4\right) .
\end{aligned}
$$

The cosine on the right side of Eq. (109) has two parts, one co-oscillating and the other counter-oscillating relative to the pre-exponential. Neglecting the co-oscillating part (its contribution averages to zero) gives

$$
G(E) \approx-i\left(\frac{t_{\phi}}{2 E}\right)^{1 / 2}\left(\frac{H}{E}\right)^{1 / 4} \sum_{n \gg 1}^{\infty} \frac{e^{i \sqrt{8 t_{\phi} E n}(1-\sqrt{H / E})}}{\sqrt{n}} .
$$

Replacing the sum over $n$ by an integral over $n$, extending the lower limit of integration to zero, and changing variables to $y=\sqrt{8 E n}$, we obtain

$$
\begin{aligned}
G(E) \approx & -i \frac{\sqrt{t_{\phi}}}{2 E}\left(\frac{H}{E}\right)^{1 / 4} \int_{0}^{\infty} d y e^{i y \sqrt{t_{\phi}}(1-H / E)} \\
= & \frac{1}{2 E}\left(\frac{H}{E}\right)^{1 / 4} \frac{1}{(1-\sqrt{H / E})}
\end{aligned}
$$

where we used $\int_{0}^{\infty} d y e^{i y}=i$. Recall that when $n$ is large, $C_{n}\left(-t_{\phi} H\right)$ contributes to the correlation amplitude $C(t)$ at large times. Therefore 
terms with large $n$ in the series representation of $G(E)$ contribute primarily to the near-energy-conserving part of $G(E)$; terms with small $n$, which we have neglected, contribute to the non-energy-conserving part of $G(E)$. Hence it is consistent for us to replace the overall factor $(H / E)^{1 / 4}$ by unity, and the term $(1-\sqrt{H / E})$ by $(1-H / E) / 2$; this yields $G(E)=1 /(E-H)$.

Now we discuss our second example; we derive from Eq. (84) the threshold behavior of the rate $-2 \operatorname{Im} R(E)$ for $E \sim 0$. Again we need focus only on large- $n$ terms on the right side of Eq. (84). For simplicity we assume that the angular momentum quantum number $l$ is zero. Assuming also that the potential is short-range (i.e., no Coulomb tail) it follows from Eqs. (84), (72), and (91) that for $E \sim 0$

$$
R(E) \sim-i|\psi(\mathbf{0})|^{2}\left(\frac{15(\pi \mu)^{3 / 2}}{t_{\phi}^{1 / 2}\left(2^{17} t_{\phi}^{3} E^{3}\right)^{1 / 4}}\right) \sum_{n \gg 1}^{\infty} \frac{e^{i \sqrt{8 t_{\phi} E n}+i \pi / 4}}{n^{9 / 4}} .
$$

Replacing the sum over $n$ by an integral over $n$ with lower limit $n_{0} \gg 1$, and changing variables to $y$ where $y^{2}=\sqrt{8 E n}$, we obtain

$$
R(E) \sim-i \sqrt{E}|\psi(0)|^{2}(\pi \mu)^{3 / 2}\left(\frac{15 e^{i \pi / 4}}{\sqrt{2} t_{\phi}^{5 / 4}}\right) \int_{y_{0}}^{\infty} d y \frac{e^{i y^{2} \sqrt{t_{\phi}}}}{y^{6}}
$$

where $y_{0}=\left(8 E n_{0}\right)^{1 / 4}$. As $E$ approaches zero, so does $y_{0}$, and the integrand becomes highly singular at the lower limit. However, before taking the limit $E \rightarrow 0$ we can regularize the integral by integrating by parts three times, and by discarding the surface terms at the upper and lower limits; the surface terms are exponentially small at the upper limit and are of order $1 /\left(y_{0} t_{0}^{1 / 4}\right)$ or less at the lower limit, and therefore negligible if $n_{0}$ is sufficiently large that $8 E n_{0} t_{0} \gg 1$. Note that the exponential $e^{i y^{2} \sqrt{t_{\phi}}}$ of Eq. (114) restricts $y$ to values less than or of order $\phi^{-1 / 2} t_{0}^{-1 / 4}$ and hence $1 /\left(y_{0} t_{0}^{1 / 4}\right)$ cannot be less than a number of order $\phi^{1 / 2}$; therefore we require $\phi \ll \pi$. After integrating by parts three times we can let the lower limit of the integral be zero, and we arrive at

$$
R(E) \sim-i \sqrt{E} t_{\phi}^{1 / 4}|\psi(0)|^{2}(\pi \mu)^{3 / 2} 2^{7 / 2} e^{i \pi / 4} \int_{0}^{\infty} d y e^{i y^{2} \sqrt{t_{\phi}}} .
$$

Performing the integration over $y$ yields, for $E \sim 0$, the threshold law

$$
-2 \operatorname{Im} R(E) \sim 2^{7 / 2}|\psi(\mathbf{0})|^{2} \pi^{2} \mu^{3 / 2} \sqrt{E},
$$


an expression which of course is independent of $t_{\phi}$. This is the correct threshold law if $l=0$ and if the potential is short-range. Indeed, as a check we can use Eqs. (18) and (79) to write, for $E \sim 0$,

$$
-2 \operatorname{Im} R(E) \sim 2^{5 / 2}(\pi \mu)^{3 / 2}|\psi(\mathbf{0})|^{2} \operatorname{Re} e^{-i 3 \pi / 4} \int_{0}^{\infty} d t \frac{e^{i E t}}{(t+\eta)^{3 / 2}}
$$

where we have introduced a positive but infinitesimal term $\eta$ in the denominator to regularize the integrand at $t=0$. Integrating the right side of Eq. (117) by parts (once), and evaluating the resulting integral, we reproduce exactly the right side of Eq. (116). For $l \neq 0$ we must modify Eq. (116) by including a factor proportional to $E^{l}$ on the right side.

If the potential has a Coulomb tail it follows from Eqs. (84), (76), and (91) that

$$
R(E) \sim|\tilde{\psi}(\mathbf{0})|^{2}\left(\frac{3 \pi^{5 / 2} Z \mu}{a_{0}\left(2^{9} t_{\phi}^{3} E^{3}\right)^{1 / 4}}\right) \sum_{n \gg 1}^{\infty} \frac{e^{i \sqrt{8 t_{\phi} E n}+i \pi / 4}}{n^{7 / 4}} .
$$

Replacing the sum over $n$ by an integral over $y$, where again $y^{2}=$ $\sqrt{8 E n}$, yields

$$
R(E) \sim 12 \pi^{5 / 2}|\tilde{\psi}(0)|^{2} e^{i \pi / 4}\left(\frac{Z \mu}{a_{0} t_{\phi}^{3 / 4}}\right) \int_{y_{0}}^{\infty} d y \frac{e^{i y^{2} \sqrt{t_{\phi}}}}{y^{4}}
$$

and, after regularizing the integral by integrating by parts twice, and subsequently integrating over $y$, we obtain

$$
R(E) \sim-i 8 \pi^{3}\left(Z \mu / a_{0}\right)|\tilde{\psi}(\mathbf{0})|^{2}
$$

which gives the energy-independent threshold law

$$
-2 \operatorname{Im} R(E) \sim 16 \pi^{3}\left(Z \mu / a_{0}\right)|\tilde{\psi}(\mathbf{0})|^{2} .
$$

As a check we can use Eqs. (21) and (79) to write, for $E \sim 0$,

$$
-2 \operatorname{Im} R(E) \sim 32 \pi^{2}\left(Z \mu / a_{0}\right)|\tilde{\psi}(\mathbf{0})|^{2} \operatorname{Im} \int_{0}^{\infty} d t \frac{e^{i E t}}{t} ;
$$

since $\int_{0}^{\infty} d t \sin (E t) / t=\pi / 2$ we reproduce Eq. (121). 


\section{Appendix C: Algorithms}

In this appendix we describe some algorithms for the numerical implementation of the series representations of $G(E)$ and $R(E)$. We consider the evaluation of (i) $C_{n}(z)$, with $z$ a linear operator, (ii) $\mathcal{I}_{n}(a)$ for all complex numbers $a$, and (iii) $e^{z}$, with $z$ a linear operator.

\section{(i) The Operator $C_{n}(z)$.}

Recall that $C_{n}(z) \equiv Q_{n}(z) e^{z}$, where $Q_{n}(z)$ is a polynomial of degree $n$ in $z$ :

$$
Q_{n}(z)=(2 z){ }_{1} F_{1}(1-n, 2,-2 z), \quad n \geq 1,
$$

with $Q_{0}(z)=1$. Using a standard recurrence relation for the confluent hypergeometric function [1] we obtain the recurrence relation

$$
(n+1) Q_{n+1}(z)=2(n+z) Q_{n}(z)-(n-1) Q_{n-1}(z), \quad n \geq 1,
$$

which can be started using $Q_{1}(z)=2 z$. Alternatively, we can formulate a backward recurrence relation for the function $B_{m}(z)$ of Eq. (62) using a standard recurrence relation for the Bessel function. [1] We find that

$$
B_{m-1}(z)=-\frac{1}{z}\left[m B_{m}(z)-2 n B_{m+1}(z)\right], \quad m \geq 1 .
$$

Since $B_{m}(z)$ decreases exponentially as $m$ increases, this backward recurrence relation can be started by putting $B_{m+1}(z)=0$ and $B_{m}(z)=1$ for a large value of $m$, and by using the identity [1]

$$
B_{0}(z)+2 \sum_{m=1}^{\infty}\left(\frac{-2 n}{z}\right)^{m} B_{2 m}(z)=1
$$

to correctly renormalize the functions. After computing the $B_{m}(z)$ we can use Eq. (61) to calculate $C_{n}(z)$.

(ii) The Integrals $\mathcal{I}_{n}(a)$.

To derive a recurrence relation for the integrals $\mathcal{I}_{n}(a)$, with $\operatorname{Im} a>0$, we start from Eq. (87). Using

$$
\cos ^{2} \theta=\left(2+e^{2 i \theta}+e^{-2 i \theta}\right) / 4
$$


we see that

$$
2 \mathcal{I}_{n}(a)-\mathcal{I}_{n-1}(a)-\mathcal{I}_{n+1}(a)=4(-1)^{n} \int_{0}^{\pi / 2} d \theta e^{i a \tan \theta-2 i n \theta} .
$$

Integrating by parts on the right side of Eq. (128), and discarding the surface term at the upper limit $\theta=\pi / 2$ (it vanishes if $\operatorname{Im} a>0$ ) yields

$$
\begin{aligned}
& 2 \mathcal{I}_{n}(a)-\mathcal{I}_{n-1}(a)-\mathcal{I}_{n+1}(a) \\
& =(-1)^{n+1}\left(\frac{2 i}{n}\right)+(-1)^{n}\left(\frac{2 a}{n}\right) \int_{0}^{\pi / 2} d \theta \sec ^{2} \theta e^{i a \tan \theta-2 i n \theta} .
\end{aligned}
$$

Recognizing that the integral on the right side of Eq. (129) is proportional to $\mathcal{I}_{n}(a)$ we arrive at the recursion formula

$$
\mathcal{I}_{n+1}(a)=(-1)^{n}\left(\frac{2 i}{n}\right)+2\left(1-\frac{a}{n}\right) \mathcal{I}_{n}(a)-\mathcal{I}_{n-1}(a), \quad n \geq 1
$$

which can be started using

$$
\begin{aligned}
& \mathcal{I}_{0}(a)=i / a, \\
& \mathcal{I}_{1}(a)=\mathcal{I}_{0}(a)+2 i e^{-a} \mathrm{E}_{1}(-a),
\end{aligned}
$$

where $E_{1}(z)$ is the exponential integral. As long as $a$ is positive (with an infinitesimal positive imaginary part) $\mathcal{I}_{n}(a)$ increases (albeit weakly) as $n$ increases, and this forward recurrence relation is stable. However, for large $a$ we have (see below) $\mathcal{I}_{n}(a) \sim(-1)^{n}(i / a)$ and therefore there is cancellation between the first and second terms on the right side of Eq. (130). Thus we consider separately, below, the case $a \gg n$.

Note that $E_{1}(z)$ has a logarithmic branch point singularity at $z=0$, and this singularity, which first appears in Eq. (132), is propagated by the recursion formula of Eq. (130) so that all terms but the first on the right-side of Eq. (84) have logarthmic branch points at $E=0$ [the branch cuts lie along the line $\arg (E)=-\phi]$. In contrast, the exact $R(E)$, which is a function of the dimensionless variable $E t_{0}$, has a square-root branch point at $E=0$ (if the potential is short-range). Presumably the converged sum on the right-side of Eq. (84) does have the correct branch-point behavior. In this regard it is helpful consider an example, namely the function $f(z)=\sum_{n=0}^{\infty}[\ln (z) / 2]^{n} / n$ !. Any approximation to $f(z)$ obtained by truncating the sum has a logarithmic 
branch point; but the infinite sum is $f(z)=\exp [\ln (z) / 2]=z^{1 / 2}$, which has no logarithmic branch point, only a square root branch point.

If $|a| \gg n$ we can represent $\mathcal{I}_{n}(a)$ by an asymptotic power series in $1 / a$. Integrating by parts on the right side of Eq. (83) yields

$$
\mathcal{I}_{n}(a)=\sum_{m=0}^{\infty}\left(\frac{i}{a}\right)^{m+1} Y_{n}^{(m)}(0),
$$

where $Y_{n}^{(m)}(\tau)$ is the $m$-th derivative with respect to $\tau$ of

$$
Y_{n}^{(0)}(\tau)=\left(\frac{\tau+i}{\tau-i}\right)^{n}
$$

We have $Y_{n}^{(0)}(0)=(-1)^{n}$ and the derivatives can be calculated using the following relation (which can be obtained after some straightforward algebra)

$$
Y_{n}^{(m+1)}(\tau)=2 n \sum_{k=0}^{[m / 2]}(-i)^{2 k+1} \frac{m !}{(m-2 k) !} Y_{n}^{(m-2 k)}(0),
$$

where $[m / 2]$ is $m / 2$ rounded to the lowest integer. It follows from Eq. (135) that $Y_{n}^{(m)}(0)$ is a real number multiplied by $i^{m}$, and hence if $a$ is real each term of the asymptotic series (133) is purely imaginary. In fact, the real part of $\mathcal{I}_{n}(a)$ is exponentially small; to see this, rotate the contour of integration on the right side of Eq. (83) by $180^{\circ}$, counterclockwise. This yields $\mathcal{I}_{n}(a)^{*}$, and since there is a pole at $\tau=i$ it follows from Cauchy's theorem that the real part of $\mathcal{I}_{n}(a)$ is $\pi i$ multiplied by the residue of this pole. Thereby we arrive at

$$
\begin{aligned}
\operatorname{Re} \mathcal{I}_{n}(a) & =e^{-a}\left(\frac{\pi}{a}\right) \sum_{m=0}^{n-1} \frac{n !(-2 a)^{n-m}}{m !(n-1-m) !(n-m) !} \\
& =-2 \pi e^{-a} \sum_{k=0}^{n-1} \frac{n !}{(k+1) !(n-k-1) !} \frac{n !(-2 a)^{k}}{k !} \\
& =-2 \pi e^{-a} L_{n-1}^{(1)}(2 a), \quad \operatorname{Im} a=0, \quad a>0 .
\end{aligned}
$$

Note again that if $a$ is real and negative, $\operatorname{Re} \mathcal{I}_{n}(a)=0$. 


\section{(iii) Exponentiation of a Linear Operator.}

To evaluate $e^{z}$ we use the Padé approximant

$$
e^{z} \approx \frac{1+\frac{z}{2}+\frac{z^{2}}{12}}{1-\frac{z}{2}+\frac{z^{2}}{12}}
$$

which matches the power series expansion of $e^{z}$ through the term in $z^{4}$. Since $z$ is an operator it is convenient to factorize this expression so that we do not incur the additional computation of $z^{2}$; thus we write

$$
e^{z} \approx \frac{\left(1-z / z_{1}\right)\left(1-z / z_{2}\right)}{\left(1+z / z_{1}\right)\left(1+z / z_{2}\right)}
$$

where the roots $z_{1}$ and $z_{2}$ are

$$
z_{1,2}=-3 \pm i \sqrt{3}
$$

If the Hamiltonian $H$ is represented by the matrix $\underline{H}$, constructed from a basis with overlap matrix $\underline{B}$, the time-evolution operator $e^{-i H t}$ becomes $e^{-i\left(\underline{B}^{-1} \underline{H}\right) t}$, and we have (letting $t \rightarrow-i t$ )

$$
e^{-H t} \approx \frac{1}{\underline{B}-\left(t / z_{1}\right) \underline{H}}\left[\underline{B}+\left(t / z_{1}\right) \underline{H}\right] \frac{1}{\underline{B}-\left(t / z_{2}\right) \underline{H}}\left[\underline{B}+\left(t / z_{2}\right) \underline{H}\right] .
$$

This is an extension of the standard Cayley form of the time-evolution operator from third- to fifth-order; we have found the stability and accuracy of exponentiation to be significantly improved by this increase in order. While it is unnecessary to calculate $\underline{B}^{-1}$ here, note that since $\underline{B}$ is real, symmetric, and positive definite it has a Cholesky decomposition [13] and hence its inverse can be calculated rapidly.

\section{Appendix D: Large- $n$ form of $c_{n}\left(t_{\phi}\right)$ when Coulomb Tail is Present}

To establish Eqs. (76) and Eqs. (77) for the large- $n$ form of $c_{n}\left(t_{\phi}\right)$ when a Coulomb tail is present, we first show how to analytically continue $c_{\mathrm{bd}, n}\left(t_{\phi}\right)$ along a path in the upper-half $t_{\phi}$-plane, from the upper edge of the cut to the positive real axis on the first sheet of the Riemann 
$t_{\phi}$-surface. To this end we seek to replace the sum over $m$ on the right side of Eq. (74) by an integral over $m$. However, the direct replacement of sum by integral is not entirely justified since the summand varies rapidly when $n \sim \infty$, and the $m$-th and $(m+1)$-th terms of the sum may differ significantly. Nevertheless, we can accomplish the passage from sum to integral by making a small modification to the integrand. We first break the cosine in the summand into a sum of two exponentials:

$$
c_{\mathrm{bd}, n}\left(t_{\phi}\right) \sim 8 \pi^{2} Z^{3}|\tilde{\psi}(\mathbf{0})|^{2}\left(\frac{2}{\pi^{2} n^{3}}\right)^{1 / 4}\left[S_{+}\left(t_{\phi}\right)+S_{-}\left(t_{\phi}\right)\right],
$$

where

$$
\left.S_{ \pm}\left(t_{\phi}\right)\right]=\sum_{m=1}^{\infty} \frac{\left(t_{\phi} E_{\mathrm{bd}, m}\right)^{1 / 4}}{2\left(m^{*} a_{0}\right)^{3}} e^{ \pm i \sqrt{8 n t_{\phi} E_{\mathrm{bd}, m}}+ \pm i \pi / 4}
$$

and we introduce the integrals

$$
I_{+}\left(t_{\phi}\right) \equiv \int_{C_{+}} d m \frac{\left(t_{\phi} E_{\mathrm{bd}, m}\right)^{1 / 4}}{2\left(m^{*} a_{0}\right)^{3}} e^{i \sqrt{8 n t_{\phi} E_{\mathrm{bd}, m}}+i \pi / 4}
$$

and

$$
I_{-}\left(t_{\phi}\right) \equiv \int_{C_{-}} d m \frac{\left(t_{\phi} E_{\mathrm{bd}, m}\right)^{1 / 4}}{2\left(m^{*} a_{0}\right)^{3}} \frac{e^{-i \sqrt{8 n t_{\phi} E_{\mathrm{bd}, m}}-i \pi / 4}}{\left(1-e^{2 i \pi m}\right)},
$$

where the contours $C_{ \pm}$run from $m=1$ to $\infty$ along the upper edge of the positive real $m$-axis (and where $m^{*}$ is now the continuous variable $m-\delta)$. We have introduced the factor $\left(1-e^{2 i \pi m}\right)$ into the denominator of the integrand of $I_{-}\left(t_{\phi}\right)$, a factor which vanishes when $m$ is an integer. The integral $I_{+}\left(t_{\phi}\right)$ is formally defined for all $t_{\phi}$ on the first sheet, except on the cut, of the Riemann $t_{\phi}$-surface, i.e., for $-\pi<\phi<\pi$, since $i \sqrt{8 n t_{\phi} E_{\mathrm{bd}, m}}=-\sqrt{8 n t_{\phi}\left|E_{\mathrm{bd}, m}\right|}$ and the exponential in the integrand of $I_{+}\left(t_{\phi}\right)$ decays as $m$ increases. Furthermore, due to this exponential, only the region in which $\left|8 n t_{\phi} E_{\mathrm{bd}, m}\right|$ is less than or of order unity contributes significantly to $I_{+}\left(t_{\phi}\right)$; in this region $m^{*}$, and hence $m$, are greater than or of the order of $\sqrt{n}$, and so the integrand varies slowly with $m$. Consequently, when $t_{\phi}$ is on the upper edge of the cut the integral $I_{+}\left(t_{\phi}\right)$ and the sum $S_{+}\left(t_{\phi}\right)$ are the same. The integral $I_{-}\left(t_{\phi}\right)$ is formally defined for all $t_{\phi}$ on the second sheet reached by crossing the branch cut, i.e., for $\pi<\phi<3 \pi$. For $t_{\phi}$ on the branch cut, the 
exponential in the numerator of the integrand of $I_{-}\left(t_{\phi}\right)$ oscillates, but it is undamped so we cannot directly replace the integral by the sum. However, we can express the integral as a sum by observing that the integrand of $I_{-}\left(t_{\phi}\right)$ has an infinite sequence of poles on the positive real $m$-axis, on the lower edge of the contour $C_{-}$, at those points where $m$ is a nonnegative integer. If we rotate $C_{-}$downwards, past the line of poles, we pick up the contributions from the poles according to Cauchy's residue theorem. The integral along the new, rotated, contour is negligible for the following reason: The exponential in the numerator of the integrand is now damped, and so the main contribution to the integral comes from the region where $m$ is greater than or of the order of $\sqrt{n}$; but the exponential $e^{2 i \pi m}$ in the denominator is exponentially large since $\operatorname{Im}(m)$ is large and negative along the important segment of the rotated contour. It follows that for $t_{\phi}$ on the branch cut, and anywhere on the second sheet, $I_{-}\left(t_{\phi}\right)$ is, when $n \sim \infty$, just the sum of the residues of the poles multiplied by $-2 \pi i$; this is the same as $S_{-}\left(t_{\phi}\right)$ when $t_{\phi}$ is on the cut.

To evaluate $I_{+}\left(t_{\phi}\right)$ we change variables from $m$ to $y=$ $\sqrt{Z e /\left(m^{*} a_{0}^{1 / 2}\right)}$. The contour of integration runs from $y=0$ to roughly $\sqrt{Z e /\left(a_{0}^{1 / 2}\right)}$. Extending the upper limit to $\infty$ (which does not affect the leading $n$-dependence of the integral) and rotating the contour of integration downwards through an angle of $\phi / 4$, replacing $y$ by $y e^{-i \phi / 4}$, gives

$$
\begin{aligned}
I_{+}\left(t_{\phi}\right) & =i\left(\frac{e^{-i \phi} \mu}{Z^{2} a_{0}}\right)\left(\frac{t_{0}}{2}\right)^{1 / 4} \int_{0}^{\infty} d y y^{4} e^{-2 y^{2} \sqrt{n t_{0}}} \\
& =\frac{i}{n^{5 / 4}}\left(\frac{3 \pi^{1 / 2} \mu}{2^{23 / 4} Z^{2} a_{0} t_{\phi}}\right), \quad-\pi<\phi<\pi .
\end{aligned}
$$

A similar change of variables for $I_{-}\left(t_{\phi}\right)$ gives, after extending the upper limit of integration to $\infty$ and rotating the contour of integration upwards through an angle of $\Theta$,

$$
I_{-}\left(t_{\phi}\right)=e^{i 5 \Theta}\left(\frac{e^{i \phi / 4} \mu}{Z^{2} a_{0}}\right)\left(\frac{t_{0}}{2}\right)^{1 / 4} \int_{0}^{\infty} d y y^{4} \frac{e^{2 y^{2} e^{i 2(\Theta+\phi / 4)} \sqrt{n t_{0}}}}{\left(1-e^{2 i \pi \delta} e^{2 i \pi\left(e^{-2 i \Theta} Z e / a_{0}^{1 / 2} y^{2}\right)}\right)} .
$$

The integral $I_{-}\left(t_{\phi}\right)$ is formally defined if $(3 \pi-\phi) / 4>\Theta>(\pi-\phi) / 4$. We set $\Theta$ equal to its minimum value $\left(\pi_{+}-\phi\right) / 4$. Let us, for the 
moment, choose $t_{\phi}$ to be on the second sheet, so that $\phi>\pi$; this implies that $\Theta<0$. Due to the exponential in the numerator on the right side of Eq. (148), the main contribution to the integral comes from values of $y$ less than or of order $1 / n^{1 / 4}$, and since $\Theta<0$ the exponential $e^{2 i \pi\left(e^{-2 i \Theta} Z e / a_{0}^{1 / 2} y^{2}\right)}$ in the denominator on the right side of Eq. (148) is negligible. It follows that

$$
\begin{aligned}
I_{-}\left(t_{\phi}\right) & \sim e^{i 5 \Theta}\left(\frac{e^{i \phi / 4} \mu}{Z^{2} a_{0}}\right)\left(\frac{t_{0}}{2}\right)^{1 / 4} \int_{0}^{\infty} d y y^{4} e^{2 y^{2} e^{i 2(\Theta+\phi / 4)} \sqrt{n t_{0}}} \\
& =-\frac{i}{n^{5 / 4}}\left(\frac{3 \pi^{1 / 2} \mu}{2^{23 / 4} Z^{2} a_{0} t_{\phi}}\right), \quad \pi<\phi<3 \pi
\end{aligned}
$$

[The right sides of Eqs. (147) and (149) differ only by a sign.] Now let us choose $t_{\phi}$ to be above the branch cut on the first sheet, so that $\phi<\pi$; this implies $\Theta>0$. Again, the main contribution to the integral comes from values of $y$ less than or of order $1 / n^{1 / 4}$, but since $\Theta>0$ the exponential $e^{2 i \pi\left(e^{-2 i \Theta} Z e / a_{0}^{1 / 2} y^{2}\right)}$ is now very large. Hence, as $t_{\phi}$ crosses the cut from below to above, moving from the second sheet to the first, $I_{-}\left(t_{\phi}\right)$ vanishes through order $1 / n^{5 / 4}$. It follows that for $t_{\phi}$ on the first sheet

$$
c_{\mathrm{bd}, n}\left(t_{\phi}\right) \sim \frac{i}{n^{2}}\left(\frac{3 \pi^{2} Z \mu|\tilde{\psi}(\mathbf{0})|^{2}}{2^{5 / 2} a_{0} t_{\phi}}\right), \quad-\pi<\phi<\pi,
$$

but that $c_{\mathrm{bd}, n}\left(t_{\phi}\right)$ vanishes through order $1 / n^{2}$ on the second sheet, i.e., when $\pi<\phi<3 \pi$. For $\phi \neq \pm \pi_{-}$we must project out those bound states for which $m^{*}$ is roughly less than $\sqrt{n}$.

We now analyse $c_{\text {cont }, n}\left(t_{\phi}\right)$ when the potential has an attractive Coulomb tail. The integral over $k$ on the left side of Eq. (70) is, strictly speaking, defined only when $\phi=0$, since $\cos \left(\sqrt{8 n t_{\phi} E_{k}}+\pi / 4\right)$ diverges as $k$ increases unless $t_{\phi}$ is real and positive. However, as before we break the cosine into a sum of two exponentials. After performing the integration, we can analytically continue the separate integrals. Let us start with $t_{\phi}=t_{0}$ and move $t_{\phi}$ along a path in the upper-half plane to the point $t_{0} e^{i \pi_{-}}$. We first change variables from $k$ to $y=(k / \sqrt{\mu})^{1 / 2}$, which removes from the integrand the branch point arising from the factor $k^{1 / 2}$. Note that $i \sqrt{8 n t_{\phi} E_{k}}$ is equal to $2 i e^{i \phi / 2} y^{2} \sqrt{n t_{0}}$, and has a negative real part over the entire path. It follows that, after breaking the cosine into a sum of two exponentials, the integral over the term in 
$\exp \left(i \sqrt{8 n t_{\phi} E_{k}}+i \pi / 4\right)$ is well-defined over the entire path since its integrand decreases exponentially as $y^{2}$ increases. On the other hand, the integral over the term in $\exp \left(-i \sqrt{8 n t_{\phi} E_{k}}-i \pi / 4\right)$ is not well-defined; its integrand increases exponentially as $y^{2}$ increases. To analytically continue this second integral we proceed as before, and rotate the contour of $y$-integration downwards through an angle $\Theta$ where $\Theta>\phi / 4$ so that the exponential does not explode with increasing $y^{2}$ as we move $t_{\phi}$. The first integral is

$$
\begin{aligned}
& \frac{1}{2} \int d^{3} k\left(t_{\phi} E_{k}\right)^{1 / 4}|\psi(\mathbf{k})|^{2} e^{i \sqrt{8 n t_{\phi} E_{k}}+i \pi / 4} \\
& =\frac{2 \pi Z \mu}{a_{0}}\left(\frac{e^{i \pi} t_{\phi}}{2}\right)^{1 / 4} \int d \Omega \int_{0}^{\infty} d y \frac{y^{4}|\tilde{\psi}(\mathbf{k})|^{2}}{1-e^{-2 \pi \gamma}} e^{2 i y^{2} \sqrt{t_{\phi} n}},
\end{aligned}
$$

where $\gamma=Z /\left(a_{0} \mu^{1 / 2} y^{2}\right)$ and where $d \Omega$ is an element of solid angle containing $\mathbf{k}$. We now change variables in the first integral from $y$ to $x=y n^{1 / 4}$ so that $k=x^{2} \sqrt{\mu / n}$ and $\gamma=\left(Z / a_{0} x^{2}\right) \sqrt{n / \mu}$; for $n \sim \infty$ we can neglect $e^{-2 \pi \gamma}$ compared to 1 , and we can replace $|\tilde{\psi}(\mathbf{k})|^{2}$ by $|\tilde{\psi}(\mathbf{0})|^{2}$, so the first integral becomes

$$
\begin{aligned}
& \frac{2 \pi Z \mu}{a_{0}} \frac{|\tilde{\psi}(\mathbf{0})|^{2}}{n^{5 / 4}}\left(\frac{e^{i \pi} t_{\phi}}{2}\right)^{1 / 4} \int d \Omega \int_{0}^{\infty} d x x^{4} e^{2 i x^{2} \sqrt{t_{\phi}}} \\
& =-\frac{i}{n^{5 / 4}}\left(\frac{3 \pi^{5 / 2} Z \mu|\tilde{\psi}(\mathbf{0})|^{2}}{2^{11 / 4} a_{0} t_{\phi}}\right) .
\end{aligned}
$$

The second integral is, after changing variables from $y$ to $y e^{-i \Theta}$,

$$
\begin{aligned}
& \frac{1}{2} \int d^{3} k\left(t_{\phi} E_{k}\right)^{1 / 4}|\psi(\mathbf{k})|^{2} e^{-i \sqrt{8 n t_{\phi} E_{k}}-i \pi / 4} \\
& =\frac{2 \pi Z \mu e^{-i 5 \Theta}}{a_{0}}\left(\frac{e^{-i \pi} t_{\phi}}{2}\right)^{1 / 4} \int d \Omega \int_{0}^{\infty} d y \frac{y^{4}|\tilde{\psi}(\mathbf{k})|^{2}}{1-e^{-2 \pi \gamma}} e^{-2 i y^{2} e^{-2 i \Theta}} \sqrt{t_{\phi} n}
\end{aligned}
$$

where now $k=\mu^{1 / 2} y^{2} e^{-2 i \Theta}$ and $\gamma=Z e^{2 i \Theta} /\left(a_{0} \mu^{1 / 2} y^{2}\right)$. We fix $\Theta$ to have its minimum value, i.e., $\Theta=\phi / 4+\eta / 2$, with $\eta$ positive but infinitesimal and change variables from $y$ to $x=y n^{1 / 4}$. For $n \sim \infty$ we have

$$
\begin{aligned}
& \frac{1}{2} \int d^{3} k\left(t_{\phi} E_{k}\right)^{1 / 4}|\psi(\mathbf{k})|^{2} e^{-i \sqrt{8 n t_{\phi} E_{k}}-i \pi / 4} \\
& \sim \frac{8 \pi^{2} Z \mu e^{-i 5 \Theta}}{a_{0}} \frac{|\tilde{\psi}(\mathbf{0})|^{2}}{n^{5 / 4}}\left(\frac{e^{-i \pi} t_{\phi}}{2}\right)^{1 / 4} \int_{0}^{\infty} d x x^{4} \frac{e^{-2(i+\eta) x^{2} \sqrt{t_{0}}}}{1-e^{-2 \pi \gamma}},
\end{aligned}
$$


where $k=x^{2} e^{-2 i \Theta} \sqrt{\mu / n}$ and $\gamma=e^{2 i \Theta}\left(Z / a_{0} x^{2}\right) \sqrt{n / \mu}$. Note that the integrand has an infinite number of poles, accumulating at $y=0$ on the line $\arg (y)=-\pi / 4$, at those points where $e^{-2 \pi \gamma}=1$. As long as $-\pi<\phi<\pi$ we have $-\pi / 2<2 \Theta<\pi / 2$, so that the contour of integration lies apart from the line of poles, and, furthermore, $\operatorname{Re} \gamma \gg 1$ so that the term in $e^{-2 \pi \gamma}$ on the right side of Eq. (154) is negligible; hence we can integrate over $x$ to give

$$
\begin{aligned}
& \frac{1}{2} \int d^{3} k\left(t_{\phi} E_{k}\right)^{1 / 4}|\psi(\mathbf{k})|^{2} e^{-i \sqrt{8 n t_{\phi} E_{k}}-i \pi / 4} \\
& \sim \frac{i}{n^{5 / 4}}\left(\frac{3 \pi^{5 / 2} Z \mu|\tilde{\psi}(\mathbf{0})|^{2}}{2^{11 / 4} a_{0} t_{\phi}}\right), \quad-\pi<\phi<\pi .
\end{aligned}
$$

Therefore, when $-\pi<\phi<\pi$ the two integrals have leading terms that are equal but opposite, and $c_{\text {cont, } n}\left(t_{\phi}\right)$ decreases faster than $n^{-2}$ as $n$ increases. However, we now let $\phi$ approach $\pi$. This forces $\Theta$ to approach $\pi_{+} / 4$, and the integration contour moves across the line of poles, to its lower edge, resulting in a rapid change in the integral by an amount equal to the contribution of the poles. To determine the value of the new integral we increase $\Theta$ further, holding $\phi$ fixed at $\pi_{+} / 4$, so that $\Theta$ moves into the range $\pi / 2<2 \Theta<3 \pi / 2$ where Re $\gamma$ is large and negative and $e^{-2 \pi \gamma}$ is exponentially large; it follows that the new integral is negligible. Hence the integral on the left side of Eq. (155) is negligible when $t_{\phi}$ lies on the second sheet. It follows that while $c_{\text {cont }, n}\left(t_{\phi}\right)$ vanishes through order $1 / n^{2}$ when $t_{\phi}$ lies on the first sheet,

$$
c_{\text {cont }, n}\left(t_{\phi}\right) \sim-\frac{i}{n^{2}}\left(\frac{3 \pi^{2} Z \mu|\tilde{\psi}(0)|^{2}}{2^{5 / 2} a_{0} t_{\phi}}\right), \quad \pi<\phi<3 \pi,
$$

when $t_{\phi}$ lies on the second sheet. We have now established Eqs. (76) and Eqs. (77).

\section{References}

[1] M. Abramowitz and I.E. Stegun, Handbook of Mathematical Functions, U.S. Dept. of Commerce, Washington D.C., 1964.

[2] R.T. Baumel, M.C. Crocker, and J. Nuttall, Phys. Rev., A 12 (1975), 486; and

R.M. Potvliege and R. Shakeshaft, J. Phys., B 21 (1988) L645. 
[3] See e.g., A.K. Bhatia and Y.K. Ho, Phys. Rev., A 41 (1990), 504; D. Wintgen and D. Delande, J. Phys., B 26 (1993), L399.

[4] P.G. Burke and K.A. Berrington, eds., Atomic and Molecular Processes: An R-Matrix Approach, Institute of Physics, Philadelphia, 1993.

[5] J. Dollard, J. Math. Phys., 5 (1964), 729.

[6] M.L. Goldberger and K.M. Watson, Collision Theory, Krieger, New York, 1975.

[7] Y. Huang, D.J. Kouri, and D.K. Hoffman, J. Chem. Phys., 101 (1994), 10493.

[8] Y. Huang, W. Zhu, D.J. Kouri, and D.K. Hoffman, Chem. Phys. Lett., 206 (1993), 96; and references therein.

[9] S. Klarsfeld and A. Maquet, Phys. Lett., A 78 (1980), 40; J. Phys., B 12 (1979), L553;

R.M. Potvliege and R. Shakeshaft, Phys. Rev., A 39 (1989), 1545.

[10] V.A. Mandelshtam and H.S. Taylor, J. Chem. Soc. Faraday Trans., 93 (1997), 847.

[11] A. Messiah, Quantum Mechanics, Vol. 2, North-Holland, New York, 1958.

[12] C. L. Pekeris, Phys. Rev., 112 (1958), 1649.

[13] W.H. Press, S.A. Teukolsky, W.T. Vetterling, and B.P. Flannery, Numerical Recipes, 2nd ed., Cambridge, New York, 1992.

[14] W.P. Reinhardt, Annu. Rev. Phys. Chem., 33 (1982), 223.

[15] R. Shakeshaft, unpublished.

[16] R. Shakeshaft, Phys. Rev., A 60 (1999), 1280; ibid, 60 (1999), 4198.

[17] H. Tal-Ezer and R. Kosloff, J. Chem. Phys., 81 (1984), 3967. 